University of Wollongong

Research Online

Australian Institute for Innovative Materials -

Papers

Australian Institute for Innovative Materials

$1-1-2016$

Azimuthal dependence of the Garton-Tomkins orbit in crossed magnetic and electric fields

Colin Bleasdale

University of Wollongong, cb123@uowmail.edu.au

Roger A. Lewis

University of Wollongong, roger@uow.edu.au

A Bruno-Alfonso

Universidade Estadual Paulista

Follow this and additional works at: https://ro.uow.edu.au/aiimpapers

Part of the Engineering Commons, and the Physical Sciences and Mathematics Commons

Research Online is the open access institutional repository for the University of Wollongong. For further information contact the UOW Library: research-pubs@uow.edu.au 


\title{
Azimuthal dependence of the Garton-Tomkins orbit in crossed magnetic and electric fields
}

\author{
Abstract \\ Work on classical closed orbits in the diamagnetic Kepler problem is predominately focused on the chaos \\ observed in the polar launch angle as opposed to the azimuthal launch angle. This is due to atomic \\ systems, along with widely studied external-field geometries (parallel magnetic and electric fields or pure \\ magnetic field), being uniform in azimuthal angle, rendering the azimuthal angle unimportant. In the case \\ of crossed magnetic and electric fields, this is no longer the case, and closed orbits do present an \\ azimuthal launch angle dependence. In atomic systems, due to their spherical symmetry, the electric-field \\ orientation in the plane perpendicular to the magnetic field does not affect the spectrum of orbits. \\ However, in shallow n-type donors in anisotropic semiconductors such as silicon, the orientation of the \\ external fields with respect to conduction-band valleys will be important. In this work we examine the \\ Garton-Tomkins orbit in crossed magnetic and electric fields, and analyze how it and its harmonics' \\ azimuthal dependencies behave through variation of the scaled field or scaled energy. At low scaled \\ fields, harmonics have either twofold or fourfold azimuthal dependencies determined by the rotational \\ symmetry of the individual harmonics. As the scaled field or scaled energy is increased, several \\ harmonics undergo significant bifurcations, resulting in large azimuthal angular regions of essentially \\ closed orbits, which will lead to strong resonances in experimental work.

\section{Disciplines} \\ Engineering | Physical Sciences and Mathematics

\section{Publication Details} \\ Bleasdale, C., Lewis, R. A. \& Bruno-Alfonso, A. (2016). Azimuthal dependence of the Garton-Tomkins orbit \\ in crossed magnetic and electric fields. Physical Review A, 94 (2), 023409-1-023409-25.
}




\title{
Azimuthal dependence of the Garton-Tomkins orbit in crossed magnetic and electric fields
}

\author{
C. Bleasdale* and R. A. Lewis ${ }^{\dagger}$ \\ Institute for Superconducting and Electronic Materials and School of Physics, University of Wollongong, \\ New South Wales 2522, Australia \\ A. Bruno-Alfonso \\ Departamento de Matemática, Faculdade de Ciências, Unesp-Universidade Estadual Paulista, \\ Bauru, São Paulo 17033-360, Brazil
}

(Received 1 April 2016; published 11 August 2016)

\begin{abstract}
Work on classical closed orbits in the diamagnetic Kepler problem is predominately focused on the chaos observed in the polar launch angle as opposed to the azimuthal launch angle. This is due to atomic systems, along with widely studied external-field geometries (parallel magnetic and electric fields or pure magnetic field), being uniform in azimuthal angle, rendering the azimuthal angle unimportant. In the case of crossed magnetic and electric fields, this is no longer the case, and closed orbits do present an azimuthal launch angle dependence. In atomic systems, due to their spherical symmetry, the electric-field orientation in the plane perpendicular to the magnetic field does not affect the spectrum of orbits. However, in shallow $n$-type donors in anisotropic semiconductors such as silicon, the orientation of the external fields with respect to conduction-band valleys will be important. In this work we examine the Garton-Tomkins orbit in crossed magnetic and electric fields, and analyze how it and its harmonics' azimuthal dependencies behave through variation of the scaled field or scaled energy. At low scaled fields, harmonics have either twofold or fourfold azimuthal dependencies determined by the rotational symmetry of the individual harmonics. As the scaled field or scaled energy is increased, several harmonics undergo significant bifurcations, resulting in large azimuthal angular regions of essentially closed orbits, which will lead to strong resonances in experimental work.
\end{abstract}

DOI: 10.1103/PhysRevA.94.023409

\section{INTRODUCTION}

The first resonances observed in atomic spectra within external fields were found in barium atoms in a magnetic field [1]. The oscillations in the spectra were linked to the chaotic motion of electrons in the plane perpendicular to the external field [2], the so-called Garton-Tomkins (hereon referred to as GT) orbit. Since this discovery, many investigations have been made in other atomic systems, mostly in hydrogen [3-10], due to its relative theoretical simplicity. The relationship between classically chaotic electron orbits and oscillating experimental atomic spectra is now well understood. The addition of a static electric field either parallel [11-15] or perpendicular (crossed) [16-24] to the magnetic field provides other systems which are solvable classically and experimentally observable. Given that atomic hydrogen is classically regular in a pure electric field [25-27] and chaotic in a pure magnetic field, the system may be observed moving between these two extremes as the ratio of magnetic to electric fields (scaled field) is varied $[11,12]$. The system in crossed fields has, until recently, been a much more complicated system to analyze theoretically due to the loss of azimuthal rotational symmetry, leading to a breakdown in traditional calculation methods. However, the recent breakthrough in the derivation of a simple classical theory for arbitrary field orientation utilizing the existing framework in simpler field geometries [28] has allowed the crossed-field system to be analyzed in a less numerically

\footnotetext{
*cb123@uowmail.edu.au

${ }^{\dagger}$ roger@uow.edu.au

†alexys@fc.unesp.br
}

intensive manner. In the case of pure magnetic fields or parallel magnetic and electric fields, the azimuthal angle is unimportant due to the rotational symmetry of the system. However, in crossed magnetic and electric fields, both polar and azimuthal angles of the system need to be considered. An additional layer of complexity can be introduced if we consider shallow $n$-type dopants in semiconductors due to the presence of conduction-band valleys. For example, in silicon, there are six equivalent conduction-band valleys. The application of a magnetic field along one of these directions reduces the symmetry. It leads to two equivalent valleys with rotational symmetry about the magnetic field direction and four equivalent valleys whose rotational axes lay on the perpendicular plane. As a result, the two sets of valleys contribute to the properties of the semiconductor. The first and only experimental results in such a system (silicon) were published seven years ago [29] and were the first observation of the so-called anisotropic diamagnetic Kepler problem (ADKP). This led to a full theoretical treatment of the effects of an anisotropic electron mass on the classical orbits of the system [30] and a numerical investigation into the experimental conditions needed to observe such an effect in a range of widely utilized semiconductors [31].

In this work, we focus on the GT orbit and its harmonics in crossed magnetic and electric fields. This is the most important and stable orbit of the system in the analysis of experimental spectra in the crossed-fields geometry [17]. The GT orbit has a polar launch angle of $\theta=90^{\circ}$ relative to the applied magnetic field, irrespective of the external field strengths (scaled field) or electron excitation energy (scaled energy). Hence, the GT orbit is an ideal candidate to analyze the azimuthal angle dependence of the system. The crossed-fields system has 
been previously investigated thoroughly for bound closed orbits (specifically, $\epsilon=-1.4$ ) [22]. A previous study [18] provided an analysis of the GT orbit's first harmonic in weak crossed fields at an energy close to the ionization threshold. We build on this work here to include higher harmonics of this orbit, a greater range of applied field strengths, and scaled energies extending above the ionization threshold. We also discuss the possibilities for extending the work in this field geometry into the semiconductor environment. For our calculations, we align the magnetic field along the $z$ axis, and the electric field along the $x$ axis. Section II provides the relevant theory for the crossed-fields system. Section III A analyzes how systematically increasing the scaled field affects the symmetries observed in different harmonics of the GT orbit and how bifurcation processes cause changes in azimuthal dependencies. Section III B focuses on systematically increasing the scaled energy, rather than scaled field, to affect the symmetries in various harmonics of the GT orbit through further bifurcation processes. Section III C analyzes some of the bifurcations observed in Sec. III A in more detail, where essentially closed orbits proliferate over large azimuthal angle ranges. Section III D focuses on the third and sixth harmonics of the GT orbit and an unusual form of bifurcation in which azimuthal dependencies reduce, rather than increase, after the bifurcation has occurred. Section IV gives a summary of the important results and conclusions.

\section{THEORY}

Recently, a generalized theory was derived for the diamagnetic Kepler problem with arbitrary external-field orientation utilizing semiparabolic coordinates [28]. In the special case of crossed magnetic and electric fields, this theoretical framework provides a much simpler method for the computation of the classical closed orbits of the system than was otherwise available. The important results for the crossed-fields system and the following numerical analysis are detailed here.

As is conventional, the equations are scaled in length

$$
\lambda=\sqrt[3]{\frac{4 \pi^{2} k e^{2}}{m \omega_{c}^{2}}},
$$

time

$$
t=T_{c} \int_{0}^{\tau} \frac{r}{\lambda} d \tau
$$

field

$$
f=\frac{4 \pi^{2} F e}{m \omega_{c}^{2} \lambda},
$$

and energy

$$
\epsilon=\frac{4 \pi^{2} E}{m \omega_{c}^{2} \lambda^{2}},
$$

where $k=1 /\left(4 \pi \epsilon_{0}\right)$ is Coulomb's constant, $\omega_{c}=e B / m$ is the cyclotron frequency, $T_{c}=2 \pi / \omega_{c}$ is the cyclotron period, $F$ is the electric field, $B$ is the magnetic field, and $E$ is the total energy of the electron. Using this scaling, and converting the system to semiparabolic coordinates, the equations of motion for crossed magnetic and electric fields are [28]

$$
\begin{aligned}
\ddot{u}= & \frac{u \epsilon}{2}-\pi^{2} u^{3} v^{2} w-\pi^{2} u v^{4}(1-w) w \\
& -\frac{f}{4} v\left(3 u^{2}+v^{2}\right) \cos (\phi), \\
\ddot{v}= & \frac{v \epsilon}{2}-\pi^{2} u^{4} v(1-w) w-\pi^{2} u^{2} v^{3} w \\
& -\frac{f}{4} u\left(u^{2}+3 v^{2}\right) \cos (\phi),
\end{aligned}
$$

and

$$
\dot{w}=\frac{f\left(u^{2}+v^{2}\right) \sin (\phi)}{4 \pi u v}+(1-2 w)\left(\frac{\dot{u}}{u}+\frac{\dot{v}}{v}\right),
$$

where $w=(d \phi / d t) / \omega_{c}$ and $\phi$ is the azimuthal angle.

The equations given in this section are explicitly for the case of an isotropic electron mass and are therefore directly applicable to either atomic systems or shallow $n$-type donors in isotropic semiconductors. While these equations do not take into account the anisotropic electron mass present in semiconductor environments such as silicon, which was detailed in previous work [30], we believe for the specific case of the magnetic field aligned along the (001) crystallographic axis, and the electric field aligned along the (100) crystallographic axis in the perpendicular plane, the results presented in this work will provide a good approximation to expected results for this system.

\section{NUMERICAL RESULTS}

In the numerical analysis, we are focusing on the GT orbit, which is the most important orbit in the analysis of experimental spectra in the crossed-fields geometry [17]. For any value of the scaled field, or scaled energy, the GT orbit has a polar launch angle of $\theta=90^{\circ}$ relative to the applied magnetic field. In the case of silicon, this is only true in the case of the magnetic field being aligned along a conduction-band valley. If the magnetic field is aligned in any other direction, the GT orbit is pulled out of the perpendicular plane due to the anisotropy of the system [30].

The conditions imposed on our numerical investigation are that the electron needs to return within a distance of $0.01 \lambda$ to the nucleus, in a time of less than $5 T_{c}$, to be considered as a closed orbit. The condition of an orbit to return within $0.01 \lambda$ to be considered as closed is somewhat arbitrary. In silicon, for example, at a magnetic field strength of $4 T, \lambda=$ $77 \mathrm{~nm}$, we are therefore concentrating on orbits that return closer than $0.77 \mathrm{~nm}$ to the nucleus, which is much closer than the effective Bohr radius of $\sim 43 \mathrm{~nm}$. The $5 T_{c}$ orbit period cutoff was chosen to coincide with the experimental results in silicon by Chen et al. [29]. Under these closed-orbit conditions, there are seven harmonics of the GT orbit present for $f=\epsilon=$ 0 , as shown in Fig. 1. Different harmonics were defined by the number of local minima present in the orbit trajectory with reference to the nucleus. At low scaled fields and energies, this is trivial; however, at high scaled fields and energies, the orbits become much more complicated and identification of different harmonics becomes increasingly difficult.

The azimuthal launch angle was sampled at $\Delta \phi=0.1^{\circ}$ intervals, and return angles were calculated as the electron 

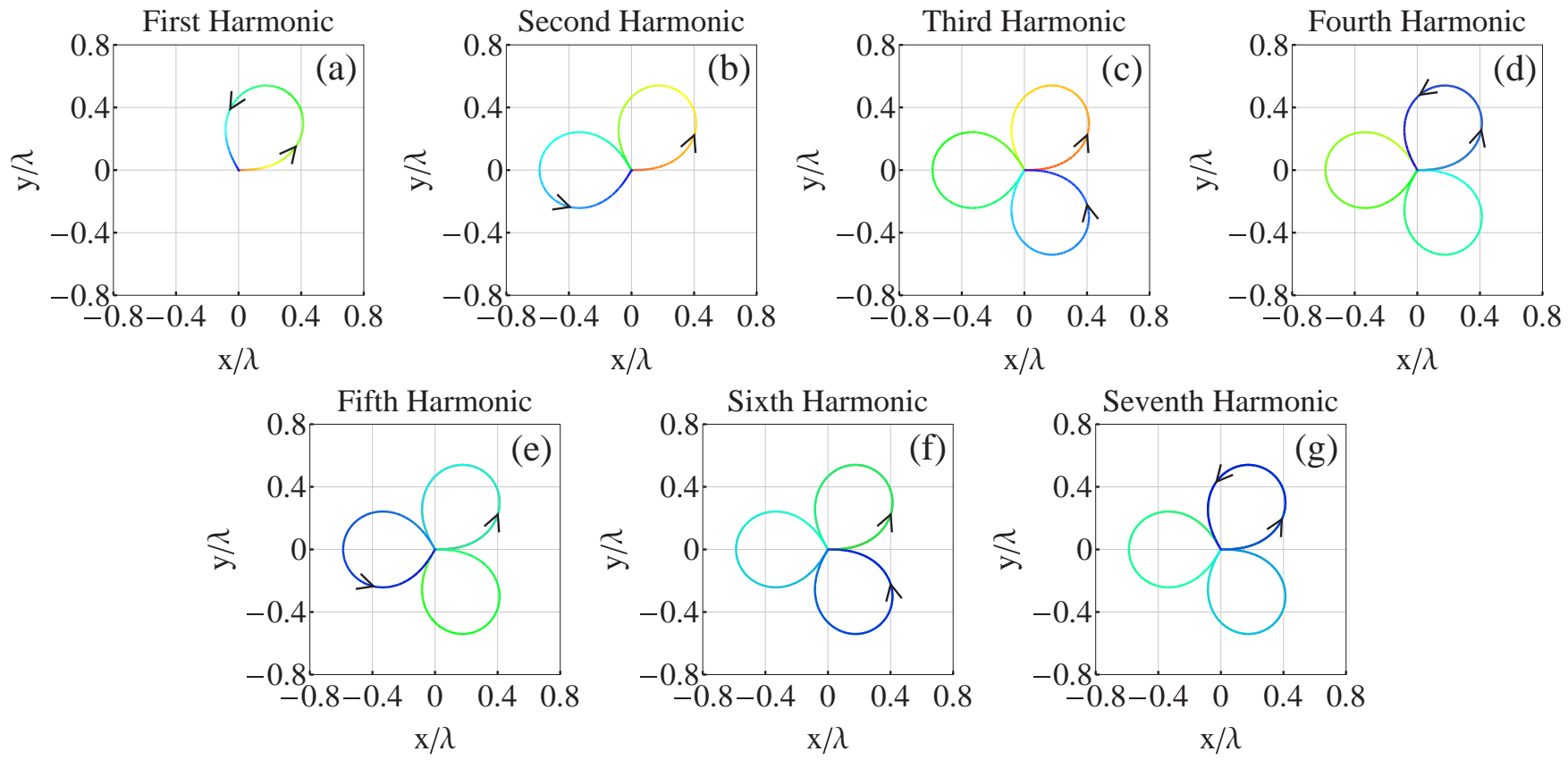

FIG. 1. The seven harmonics of the GT orbit for $f=\epsilon=0$ and $\phi=0^{\circ}$. The color of the orbit evolves with time from launch (red) to return (blue) as denoted by the direction of the arrows, which show the launch and return branches of the orbit path. The beginning of the orbit is not visible after the third harmonic due to the overlap as it undergoes a greater than $360^{\circ}$ rotation about the $z$ axis. The periods of these harmonics are successive multiples of $2 T_{c} / 3$.
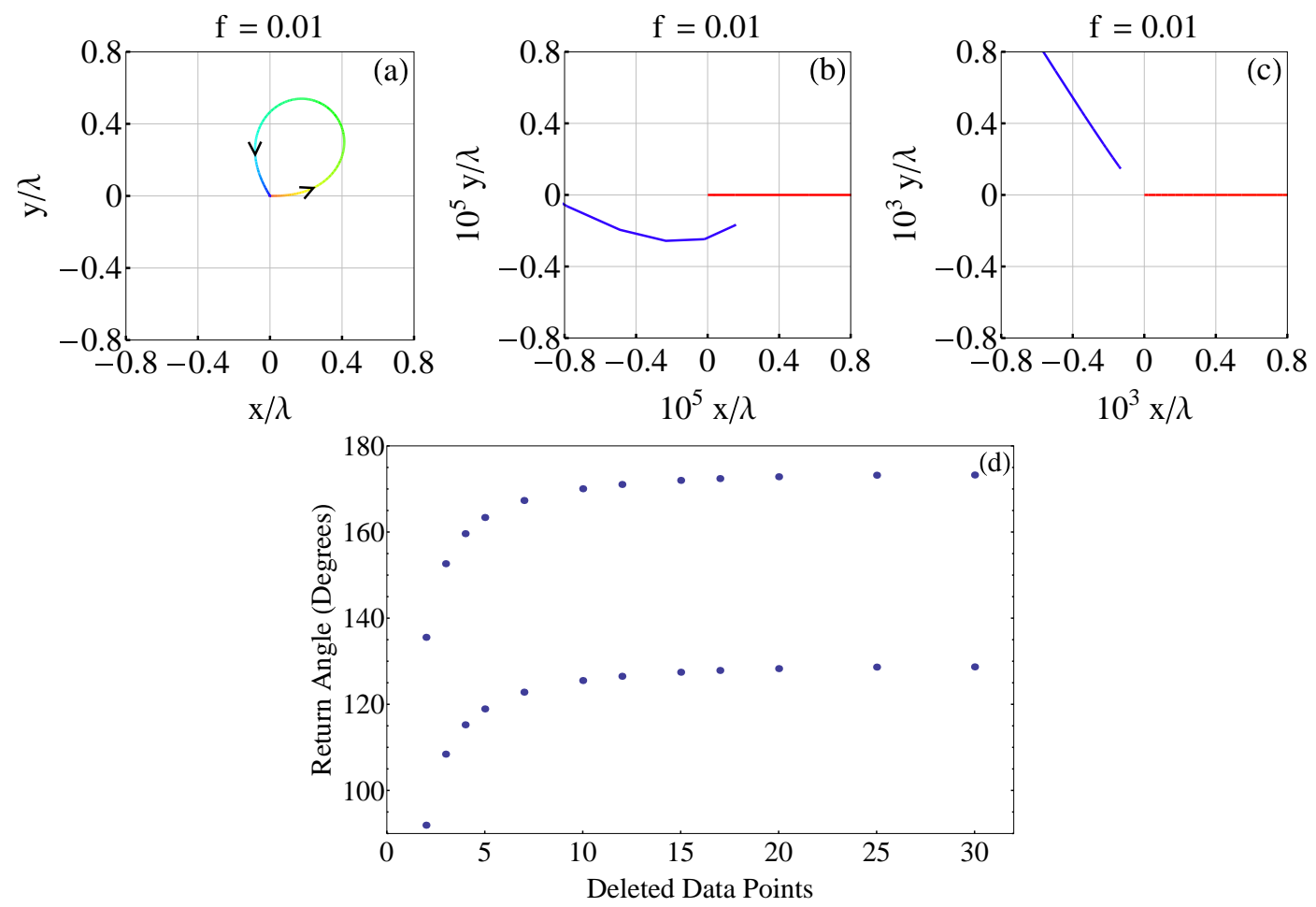

FIG. 2. Panels (a), (b), and (c) show an example of a seemingly closed orbit for $f=0.01, \epsilon=0$, and $\phi=0^{\circ}$. The color of the orbit evolves with time from launch (red) to return (blue) as denoted by the direction of the arrows which show the launch and return branches of the orbit path. Panel (a) is the entire orbit, panel (b) zooms in on the region around the nucleus to reveal the problem with using the closest point for calculating the return angle. Panel (c) shows the orbit minus the last nineteen points to reveal a good estimate of the return angle. Note panel (b) is magnified two orders of magnitude further than panel (c). Panel (d) shows the calculated return angle as a function of the number of data points omitted from the closest pass to the nucleus. Two orbits of high curvature near the nucleus from Fig. 9 were considered. After $\sim 20$ points from the nucleus, the return angle stabilizes and was therefore utilized as our condition for the calculation of the return angle. 

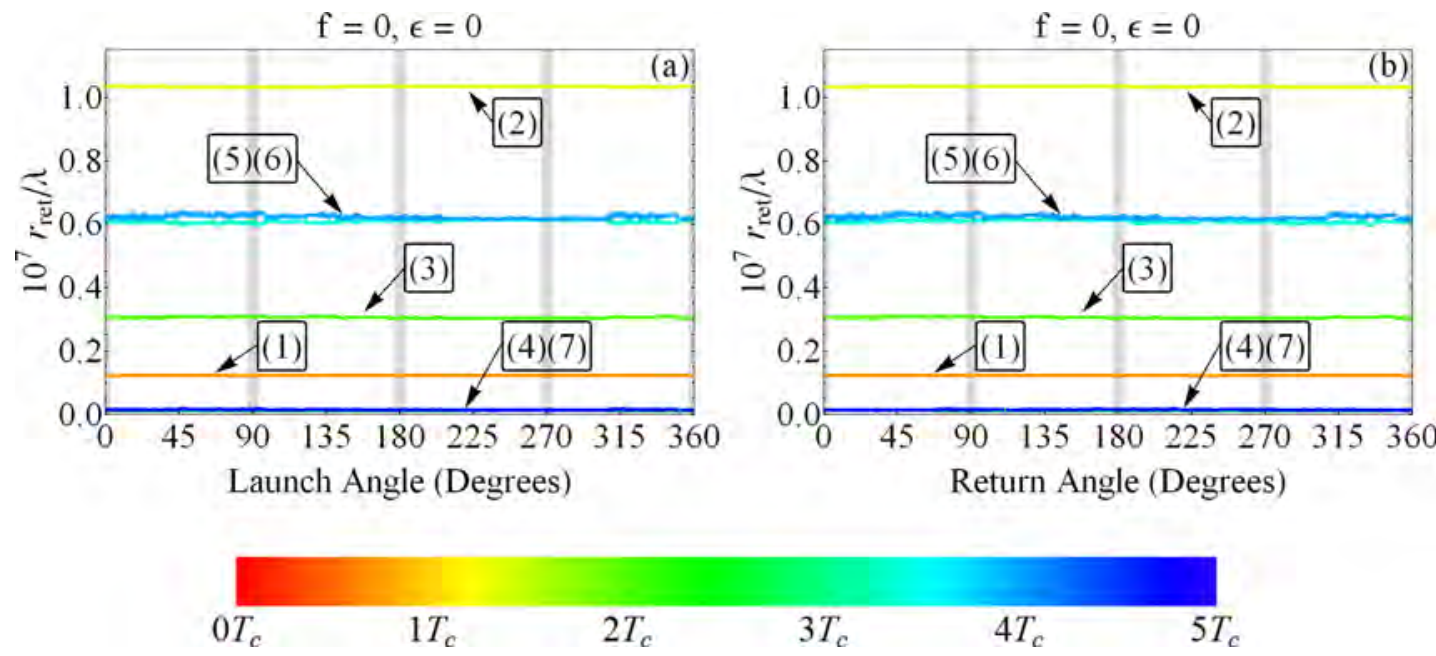

FIG. 3. Return distance $\left(r_{\text {ret }}\right)$ to the nucleus as a function of azimuthal (a) launch and (b) return angle for $f=\epsilon=0$. Data points are shaded according to their orbital period in the range 0 to $5 T_{c}$ as indicated by the bar at the bottom of the figure. This convention will be adopted throughout the paper. Vertical grid lines represent the angles of $0^{\circ}, 90^{\circ}, 180^{\circ}$ and $270^{\circ}$ with respect to the electric-field direction, on the plane perpendicular to the magnetic field. The bracketed numbers represent the harmonic numbers as identified in Fig. 1.

azimuth at the twentieth-last sample point of a given loop of its trajectory. This choice is due to the majority of orbits in this system, at finite scaled fields and energies, not being completely closed. Therefore, the closest point to the nucleus may lie in a different angular region to the general return direction of the orbit. We found, through analyzing orbits whose curvature was high around the nucleus, that the omission of the final nineteen points was sufficient to minimize this effect, as seen in Fig. 2. The return angles should therefore be seen as good approximations, rather than exact quantities, and are more accurate for orbits that return closest to the nucleus. Figures 2(a), 2(b), and 2(c) show an example of this phenomenon where the final point would yield a return angle of $\phi \sim 315^{\circ}$ rather than the $\sim 120^{\circ}$ expected.

\section{A. Scaled field dependence of launch and return angles}

In this section, we systematically increase the scaled field, at a fixed scaled energy of $\epsilon=0$, to investigate how the azimuthal dependencies of the first seven harmonics of the GT orbit evolve in crossed magnetic and electric fields.

We first consider the case of magnetic field alone. Figure 3 shows the azimuthal dependence of both launch and return angles for the GT orbit and its harmonics under pure magnetic-field conditions $(f=0)$. There is no azimuthal angle dependence in this case, as expected, given the rotational symmetry of the system in a pure magnetic field. The variation in return distance $\left(r_{\text {ret }}\right)$ for the different harmonics is an artifact of the numerical procedure rather than a real physical effect. However, considering the scale on the left axis, all harmonics return very close to the nucleus and in the following numerical results hereafter, this effect is negligible as the scale increases.

We now introduce a small electric field along the $x$ axis perpendicular to the applied magnetic field. Figure 4 shows the azimuthal dependence of both launch and return angles of the GT orbit with a small crossed electric field of $f=0.01$ applied in the plane perpendicular to the magnetic field along $\phi=0^{\circ}$. This small perturbation is enough to break the
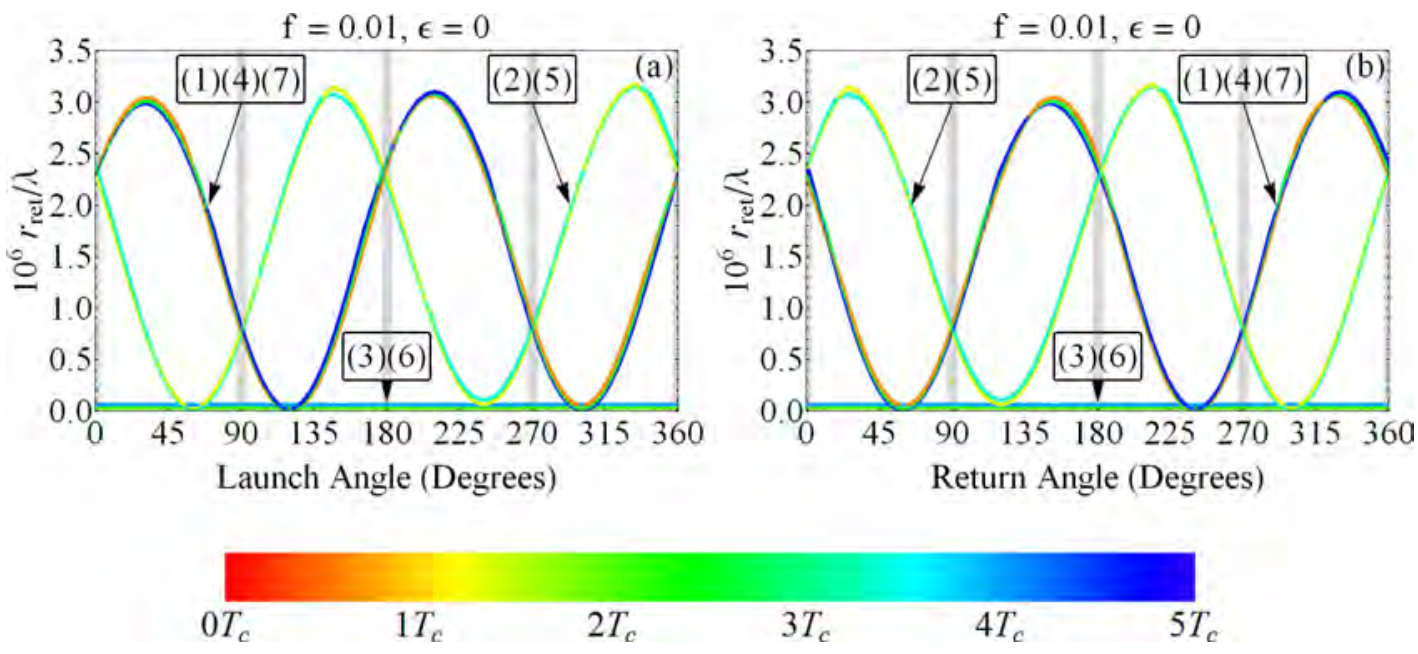

FIG. 4. Return distance $\left(r_{\text {ret }}\right)$ to the nucleus as a function of azimuthal (a) launch and (b) return angle for $f=0.01$ and $\epsilon=0$. 

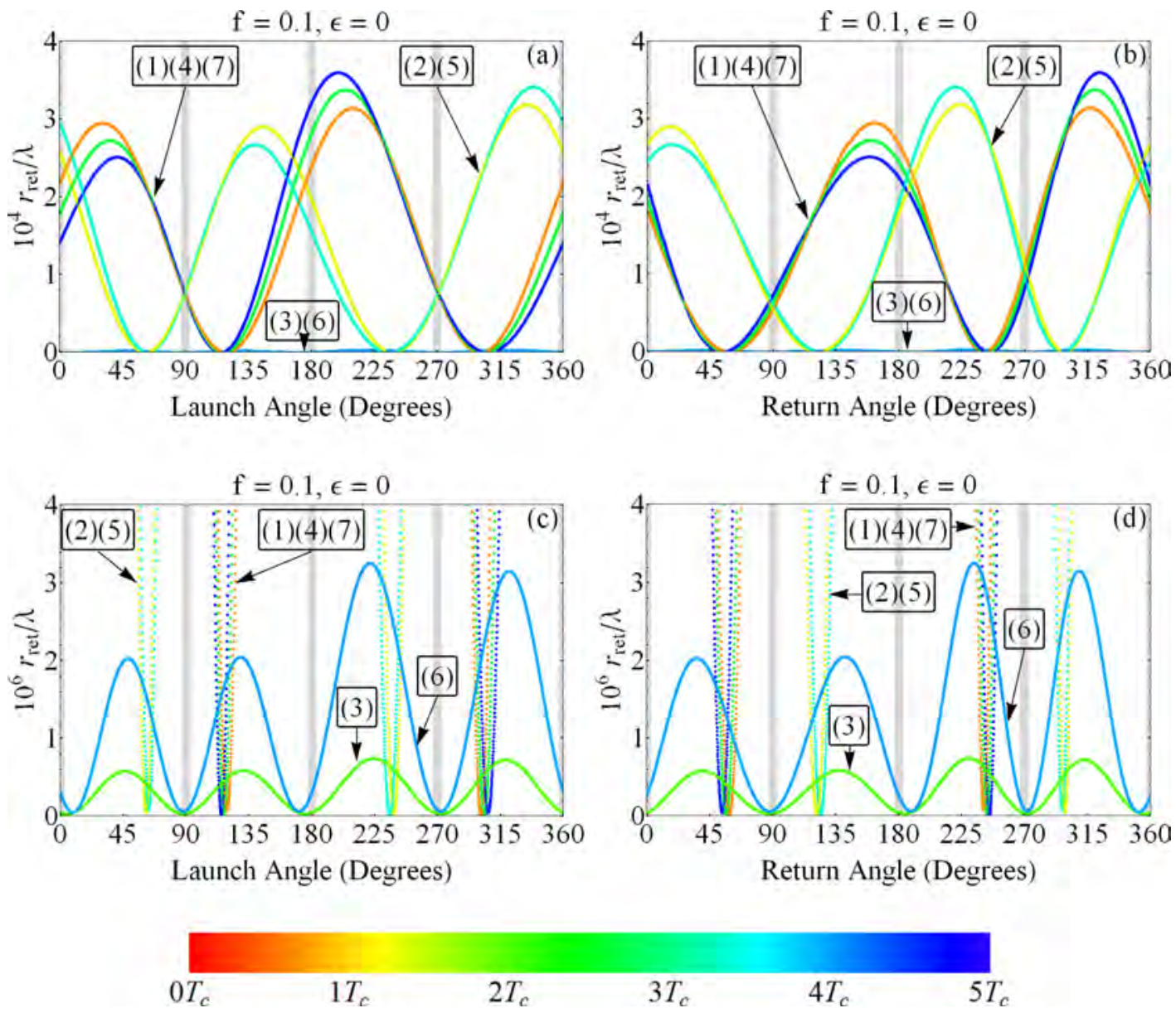

FIG. 5. Return distance $\left(r_{\text {ret }}\right)$ to the nucleus as a function of azimuthal launch (left column) and return (right column) angle for $f=0.1$ and $\epsilon=0$. The second row is a magnification of the first row to reveal the relatively weak azimuthal angle dependencies of the third and sixth harmonics.

rotational symmetry of the system and a twofold azimuthal angle dependence appears in both the launch and return angles, corresponding to orbits returning with or against the electric field. This is in accordance to what was observed in bound closed orbits [22]. Interestingly, the third and sixth harmonics show no azimuthal dependence. Intuitively, one would expect these harmonics to show at least some azimuthal dependence, especially given their periods are longer than the first and second harmonics. These azimuthal-angle independent orbits are essentially periodic and have a threefold rotational symmetry about the $z$ axis (as shown in Fig. 1); therefore, the net effect of the electric field along them is negligible.

From the scale on the left side of Fig. 4, which is of the order of $10^{-6} \lambda$, we can see that the azimuthal angle dependence is relatively weak as the entire $360^{\circ}$ range contains closed orbits. Interestingly, the return distance for the azimuthal-dependent harmonics is nearly identical for different harmonics of similar return angle in Fig. 1. In regards to the minima of the return angle of the azimuthal-dependent harmonics, the closest passes to the nucleus occur at azimuthal angles which fall away from the angles at $\phi=0^{\circ}, 90^{\circ}, 180^{\circ}$, and $270^{\circ}$, on the plane perpendicular to the magnetic field. One may then expect the azimuthally independent harmonics to contribute the strongest oscillations to the spectrum. However, this would be a false conclusion, given that the lower harmonics have a much stronger stability under variation of the polar launch angle.

While not studied in detail within this work, recurrence strengths observed from closed orbits in atomic systems, and those in an anisotropic environment such as silicon, may differ due to the presence of six conduction-band valleys. In atomic systems for the case of $f=0$, the recurrence peak heights decrease with increasing harmonics, due to their corresponding polar angle dependencies.

The azimuthal dependence observed in non-rotationallysymmetric orbits gives an insight into how resonances in the crossed-field geometry are weaker than those in rotationallysymmetric systems $[18,21]$. In such cases, every closed orbit is part of a large family of closed orbits sharing the same polar launch angle but different azimuthal launch angles. In the crossed-fields case, due to the loss of rotational symmetry, this is no longer the case, as is seen in the azimuthal dependencies obtained in our calculations.

We now increase the electric field further. Figure 5 shows the azimuthal angle dependence of launch and return angles with the scaled field raised by an order of magnitude to $f=0.1$. The azimuthal-dependent harmonics from Fig. 4 show virtually the same twofold launch and return azimuthal angle dependence as before, except now on a much larger scale of $10^{-4} \lambda$. Interestingly, with the increase in scaled field 

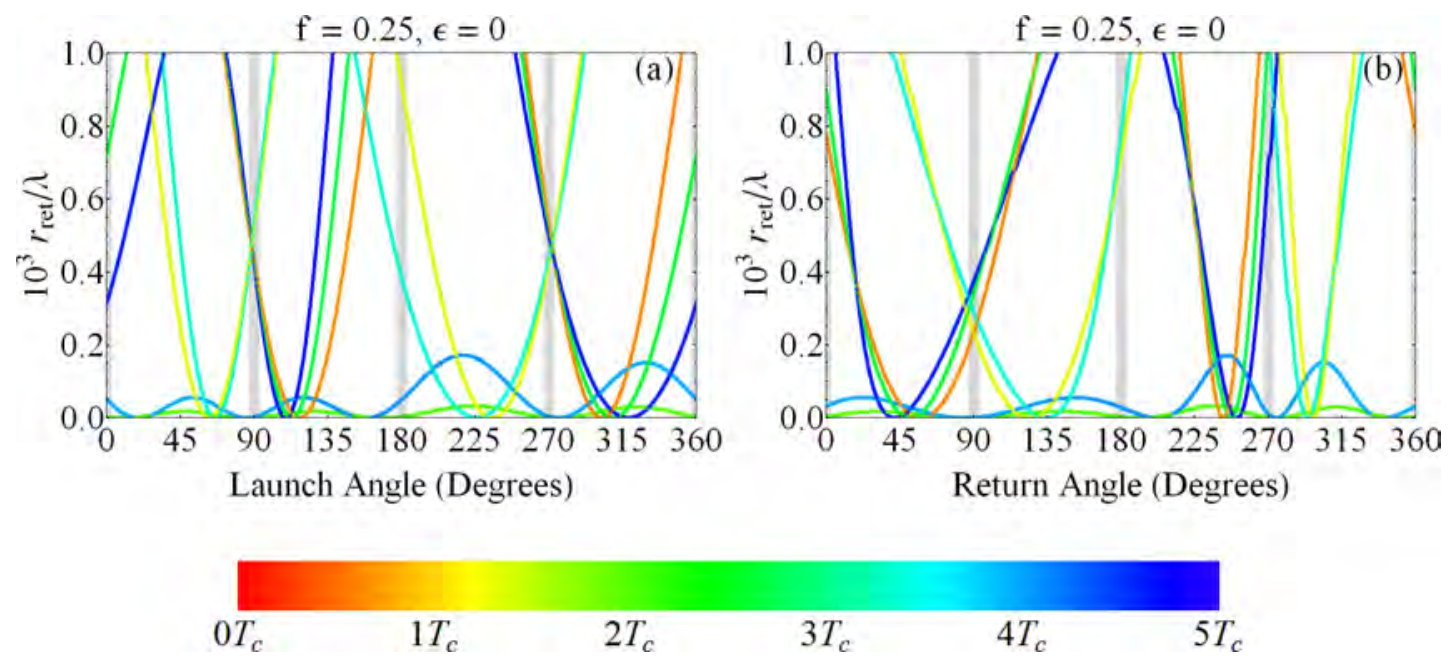

FIG. 6. Return distance $\left(r_{\text {ret }}\right)$ to the nucleus as a function of azimuthal (a) launch and (b) return angle for $f=0.25$ and $\epsilon=0$. Due to the growing complexity of the figures being presented, harmonic labels will not be included, but may still be identified through the shading based on the orbit period.

leading to a stronger perturbation away from the rotational symmetry at zero field, the peaks alternate between shortest and longest harmonics returning closer to the nucleus. The azimuthal-independent harmonics from Fig. 4 appear to continue showing no azimuthal angle dependence until we zoom in to reveal a very small fourfold launch and return azimuthal dependence in Figs. 5(c) and 5(d). We attribute this fourfold azimuthal dependence to these orbits having an approximate rotational symmetry about the $z$ axis. The field has perturbed these harmonics enough from this rotational symmetry that every pass of either the $x$ or $y$ axis yields a shift from being predominantly with or against the electric field, leading to the fourfold azimuthal angle dependence. In the first, fourth, and seventh harmonics, the minima around $\phi=45^{\circ}$ in return angle have become slightly more stable relative to the minima around $\phi=225^{\circ}$. To be concrete, an orbit is more stable than another if it has a larger number of neighboring orbits which are considered as closed orbits. The number of neighboring orbits is, of course, dependent on the sampling step size of the calculations, as realistically, there is a continuous spectrum of orbits which cannot be enumerated. However, for a constant step size in azimuthal angle, an increase in stability is identified in our calculations as a widening of the parabolic dispersion at $\phi=$ $45^{\circ}$ return angle relative to the dispersion centered at $\phi=225^{\circ}$. A similar behavior is also observed for the second and fifth harmonics. It is interesting to note that the launch and return angles of the third and sixth harmonics in Figs. 5(c) and 5(d) occur near the angles $\phi=0^{\circ}, 90^{\circ}, 180^{\circ}$, and $270^{\circ}$ with respect to the external-electric-field orientation. We expect this to lead to different relative peak heights in experimental results between these harmonics and the others than observed at $f=0$.

Increasing the scaled field further, Fig. 6 shows the azimuthal angle dependence of launch and return angles for a scaled field of $f=0.25$. The azimuthal dependencies have grown so much that there are angles where the azimuthaldependent harmonics of Fig. 4 are no longer considered as containing closed orbits. The relative stability of the third harmonic, especially, is still very strong, as indicated by its small azimuthal dependence. The increase in scaled field leads to some minima positions beginning to shift. Specifically, for the azimuthal-dependent harmonics in Fig. 4, the longer-period harmonics shift their minima away from the minima of the shorter-period harmonics and towards the electric-field orientation. A different effect is observed in the third and sixth harmonics, with the latter shifting the two minima at $\phi=0^{\circ}$ and $\phi=180^{\circ}$ away from the minima it shared with the third harmonic at lower fields. However, the other two minima at $\phi=90^{\circ}$ and $\phi=270^{\circ}$ remain tied to the third harmonic. The increase in stability of return-angle minima in the $\phi=0-180^{\circ}$ range over minima in the $\phi=180-360^{\circ}$ range has also become more pronounced with the increase in scaled field.

Figures 7(a) and 7(b) show the azimuthal angle dependence of launch and return angles for a scaled field of $f=0.5$. The electric field strength has perturbed the system to the extent that harmonics previously seen as weakly azimuthally dependent now show a strong azimuthal dependence, leading to a more complicated picture of the system. Due to the complicated nature of these figures, and subsequent figures at higher scaled fields, it is more useful to separate harmonics who shared the same return angle at zero scaled field into different figures to allow an easier analysis of the system. These are shown in Figs. 7(c)-7(h).

Figures 7(c) and 7(d) show the launch and return azimuthal dependencies of the first, fourth, and seventh harmonics. The longer-period harmonics become increasingly separated from the first harmonic as the scaled field is increased and the minima of the seventh harmonic is now closest to the electricfield orientation. The difference in stability between the two minima of the same harmonic is also further accentuated with the seventh harmonic showing a significant increase in stability over the shorter-period harmonics.

In Figs. 7(e) and 7(f) the second and fifth harmonics are shown. The opposite behavior is observed in these harmonics than was observed for the first, fourth, and seventh harmonics. The fifth harmonic is being drawn away from the electricfield orientation. As seen for the seventh harmonic, as it is being drawn away from the second harmonic by the increasing electric field, the stability increases. 

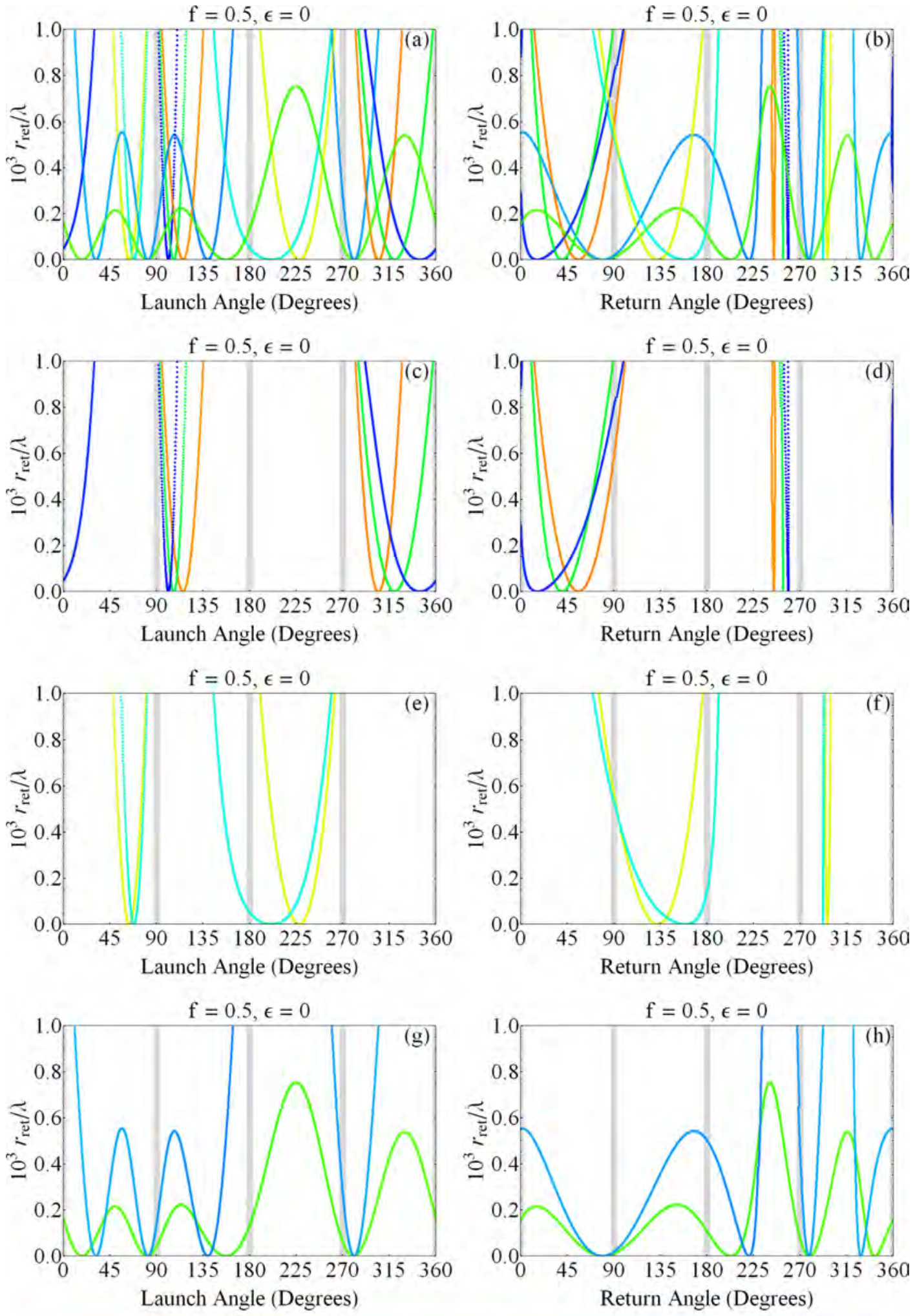
$0 T_{c} \quad 1 T_{c}$
$2 T_{c}$
$3 T_{c}$
$4 T_{c}$
$5 T_{c}$

FIG. 7. Return distance $\left(r_{\text {ret }}\right)$ to the nucleus as a function of azimuthal launch (left column) and return (right column) angle for $f=0.5$ and $\epsilon=0$. Due to the complexity of panels (a) and (b), further panels are given for harmonics who have the same return angle at low scaled fields to gain better insight into the evolution of the system. The second row shows the first, fourth, and seventh harmonics, the third row shows the second and fifth harmonics, and the fourth row shows the third and sixth harmonics. 

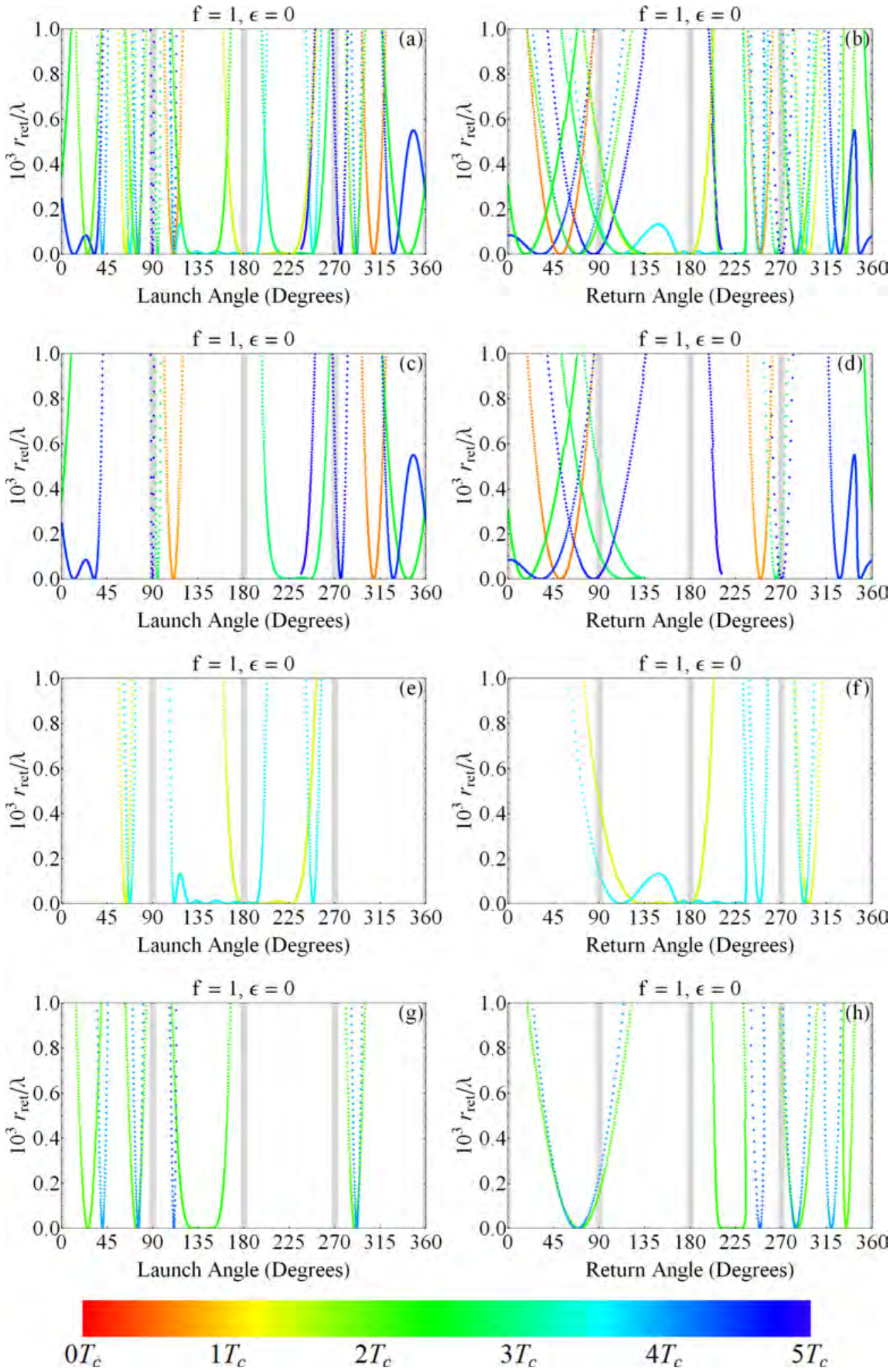

FIG. 8. Return distance $\left(r_{\text {ret }}\right)$ to the nucleus as a function of azimuthal launch (left column) and return (right column) angle for entire set of harmonics and comparable harmonics at $f=1$ and $\epsilon=0$.

Figures 7(g) and 7(h) show the third and sixth harmonics which, at low fields, showed no azimuthal dependence. Here the sixth harmonic shows the strongest azimuthal dependence, as one would expect, given its longer orbit period. The minima positions of these harmonics have diverged from each other around $\phi=0$ and $180^{\circ}$ but remain more closely tied together at $\phi=90$ and $270^{\circ}$.

Doubling the field now, Fig. 8 shows the azimuthal angle dependence of launch and return angles for both the entire set of harmonics and selected harmonics at $f=1$. Here the 

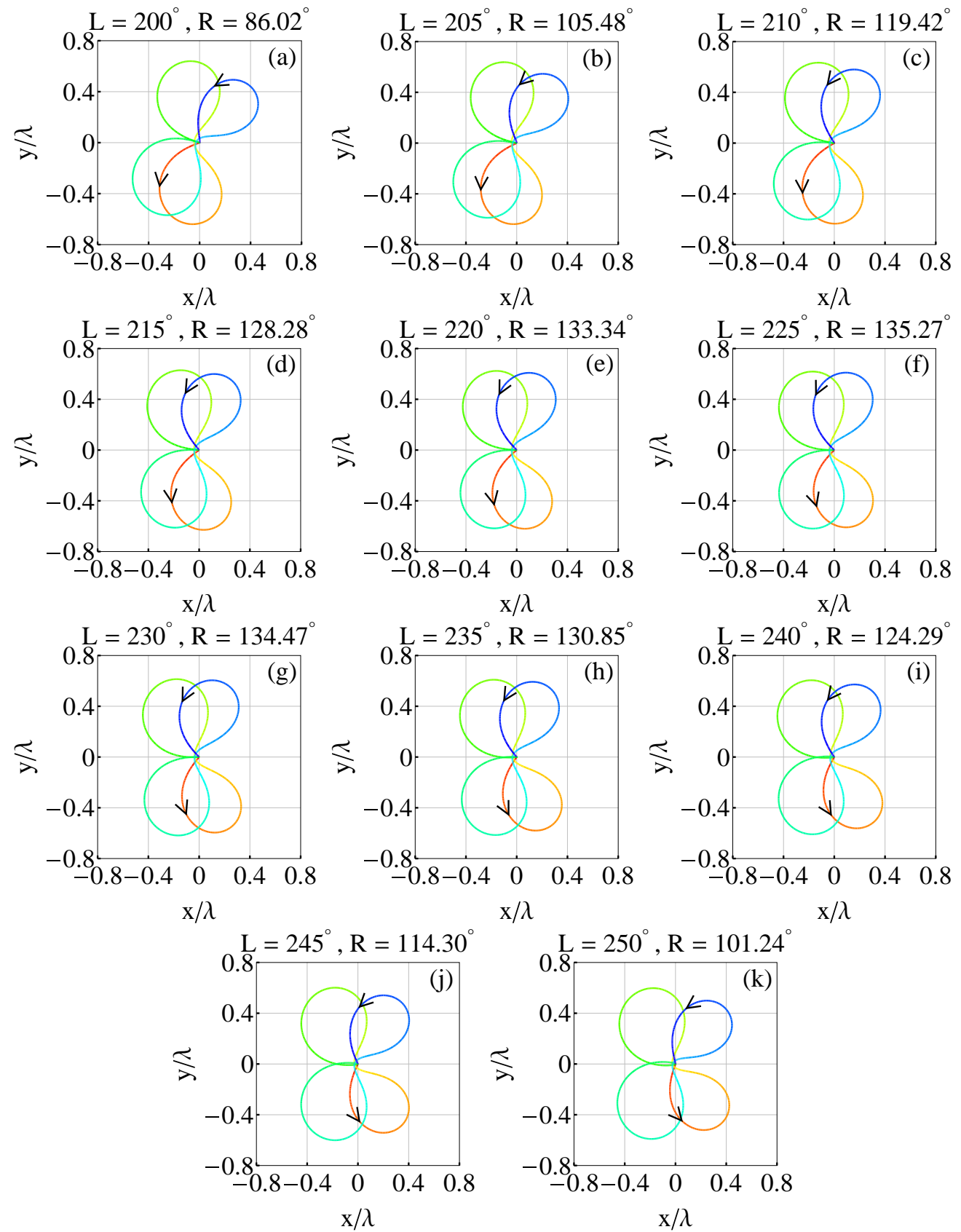

FIG. 9. Variation of the fourth harmonic as the launch angle ("L" in figure titles) is increased from $\phi=200$ to $250^{\circ}$ in $5^{\circ}$ increments. The color of the orbit evolves with time from launch (red) to return (blue) as denoted by the direction of the arrows which show the launch and return branches of the orbit path. The return angle ("R" in figure titles) increases with launch angle until $\phi=225^{\circ}$, after which it decreases with increasing launch angle leading to the unusual feature present in Fig. 8(d).

complexity of the results has increased and harmonics sharing the same return angle at zero scaled field need to again be considered separately. At this high scaled field, the relationship between comparable harmonics has been essentially broken. This can be seen most readily in Figs. 8(c) and 8(d), where there is no longer a resemblance between harmonics previously observed to be similar in nature. The first harmonic here remains relatively unchanged from earlier figures; however, the fourth and seventh harmonics have broken their twofold symmetry with new minima presenting themselves in the results. There is a discontinuity in the calculations due to orbits breaking the $5 T_{c}$ threshold. An unexpected feature presents itself in the fourth harmonic between $\phi=200$ and $250^{\circ}$ launch angle and $\phi=90$ and $135^{\circ}$ return angle. In the return angle, rather than the standard parabolic dispersion in return distance with azimuthal angle, the curve folds back, almost upon itself, once reaching the minimum. In Fig. 9, a series of fourth harmonic orbits with increasing launch angle in this region show this is not an artifact of the return-angle calculation process but rather a real effect due to the relative strength of the electric field.

Figures 8(e) and 8(f) show the second and fifth harmonics where large angular regions of essentially closed orbits now dominate the azimuthal spectrum. This resembles the so-called 
'orbit mixing' observed in Ref. [28] and is characteristic of a bifurcation process which leads to the breaking of previously held azimuthal symmetries. A finer mesh of scaled fields in the region from $f=0.5-1$ (presented in Sec. III C) shows these large angular regions were also present before the breaking of symmetries in the fourth and seventh harmonics in Figs. 8(c) and 8(d). These bifurcations may be experimentally observable, as bifurcation processes have been in other field configurations in atomic systems. Furthermore, the scaled fields in which this is being observed in the crossed-fields system are similar to those found for the parallel-fields geometry [28].

Conversely to the other harmonics, Figs. 8(g) and 8(h) show the third and sixth harmonics retaining their fourfold symmetry. However, their minima have shifted away from their low field positions completely. Perhaps surprisingly, minima which where shared between these harmonics at lower scaled fields are still present even at this high scaled field.

Increasing the field further, Fig. 10 shows the azimuthal angle dependence of launch and return angles for both the entire system and selected harmonics at $f=1.5$. As at $f=1$, the system is very complicated and harmonics sharing the same return angle at zero scaled field need to be considered separately. Concentrating on the first, fourth, and seventh harmonics in Figs. 10(c) and 10(d), the fourth harmonic is showing an increased stability as it aligns itself with the external electric field. Given we have moved from low to high scaled fields, the first harmonic has remained relatively unchanged, in that it has retained its twofold azimuthal dependence and its minima remain close to their original positions at low scaled field.

In the second and fifth harmonics shown in Figs. 10(e) and 10(f), the fifth harmonic still presents a large angular area of stability in the return angle. The second harmonic now shows a clear fourfold azimuthal dependence at this high scaled field due to the bifurcation seen occurring at $f=1$. The fifth harmonic shows a much higher degree of azimuthal dependency, indicating it has undergone many bifurcations.

In Figs. $10(\mathrm{~g})$ and $10(\mathrm{~h})$, the third and sixth harmonics, which up until $f=1$ had held their original fourfold symmetry, now display sixfold and eightfold azimuthal dependencies, respectively, indicating they have undergone many bifurcation processes in moving from $f=1-1.5$.

Over the course of Sec. III A, we have shown the return distance as a function of both launch and return angles separately. While this is insightful for investigating the azimuthal dependencies of the harmonics, the relationship between launch and return angles is difficult to access. In Fig. 11, we show the return angle as a function of launch angle as the scaled field is increased from $f=0-1.5$. As is expected at $f=0$, due to the rotational symmetry of the system, there is a linear relationship between launch and return angles. However, as the scaled field is increased, and the rotational symmetry is broken, the linear relationship between launch and return angles is also broken. Discontinuities begin to appear in the curves at $f=0.25$ due to orbits not returning within $0.001 \lambda$ of the nucleus. Moving to higher scaled fields, the gradients of the lines vary substantially. A steep gradient would indicate greater chaos in the orbit, as a small change in initial launch angle results in a large variation in return angle. Conversely, a shallow gradient would indicate an element of stability in the orbits, as a small variation in return angle is observed for large variations in initial launch angle. Interestingly, at high scaled fields, the closed orbits seem to congregate along the diagonals of the figures. Orbits which arrange themselves along the diagonal defined by equal launch and return angles correspond to orbits with high rotational symmetry about the magnetic field, which we have shown to be more stable to variations in scaled field in earlier figures.

\section{B. Scaled energy dependence of launch and return angles}

In this section, we focus on fixing the scaled field and varying the scaled energy. The purpose is to investigate how increasing the scaled energy affects the symmetries of the first seven harmonics of the GT orbit. The scaled energy is varied in integer steps over the region $\epsilon=0-4$ as was utilized in our previous work [28]. This region of scaled energy has been shown to be important in the analysis of experimental results published in silicon $[29,30]$. The scaled field is taken at fixed values of $f=0.25$ and 0.5 , as these scaled fields showed interesting features in the calculations.

Figures 12 and 13 show the launch and return azimuthal dependencies for the seven harmonics of the GT orbit as the scaled energy is incrementally increased for a fixed scaled field of $f=0.25$. As was shown in our previous work, increasing the energy in such a way affects the periods of the orbits substantially [28]. This can be observed in these figures as changes in shading as the scaled energy is increased. The sixth and seventh harmonics disappear from the calculations altogether at $\epsilon=3$ and $\epsilon=1$ respectively, as they now exceed $5 T_{c}$. The first and fourth harmonics are shown on the left columns of these figures. The first harmonic showed very little change when increasing the scaled field; however, with increasing scaled energy, the launch and return angle minima are shifting toward $\phi=90$ and $270^{\circ}$. This effect is not observed in Sec. III A and points to the scaled energy being a more effective tool in shifting the minima of these orbits with respect to the external electric-field orientation.

The fourth harmonic undergoes another form of bifurcation with increasing scaled energy between $\epsilon=2$ and $\epsilon=4$, moving from a twofold to fourfold azimuthal dependence. At $\epsilon=3$, a large azimuthal region of essentially closed orbits exists which is an indication of a bifurcation process taking place. One would expect this feature to cause an increase in experimental recurrence peak height at this scaled energy for this harmonic. Therefore, in the crossed-fields system, bifurcations occur either with varying scaled field or scaled energy.

The middle columns of Figs. 12 and 13 show the second and fifth harmonics' evolution with increasing scaled energy. In this scaled-energy range, both harmonics undergo bifurcations and move from twofold to fourfold azimuthal dependencies. The fifth harmonic, having the longest period, is more susceptible to changes in the system. Therefore, its bifurcation occurs at a lower value of $\epsilon=1$ than the second harmonics' at $\epsilon=3$. The fifth harmonic also shows the potential for another bifurcation at scaled energies exceeding $\epsilon=4$. Both bifurcations in the two harmonics occur over large launch and return azimuthal angle regions, and therefore will have a significant effect in experimental data. 

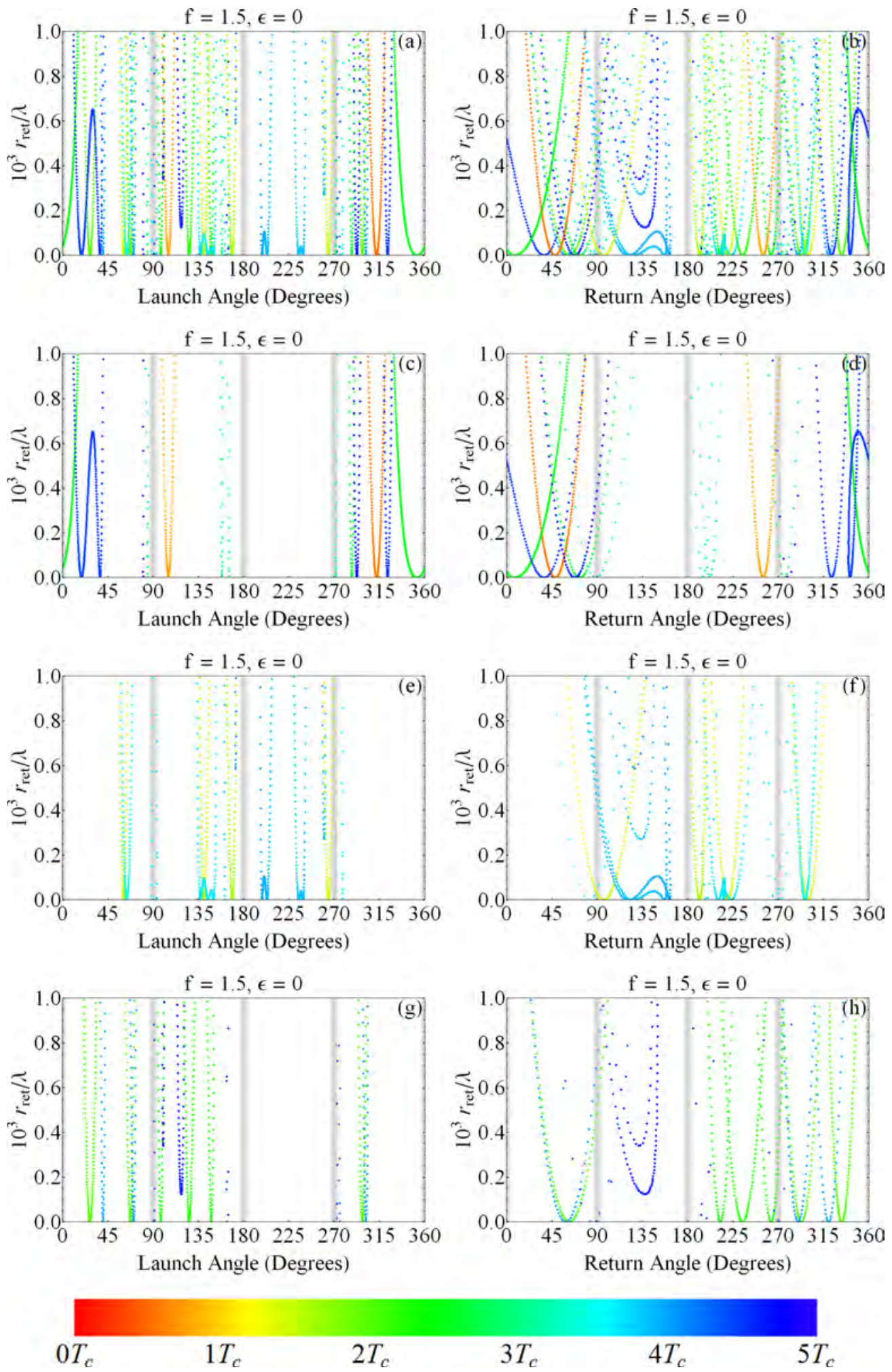

FIG. 10. Return distance $\left(r_{\text {ret }}\right)$ to the nucleus as a function of azimuthal launch (left column) and return (right column) angle for entire system and harmonics who share the same return angle at low scaled fields at $f=1.5$ and $\epsilon=0$.

The right columns in Figs. 12 and 13 show the third and sixth harmonics and their evolution with increasing scaled energy. Interestingly, these harmonics undergo a bifurcation between $\epsilon=0-1$; however, they move from a fourfold azimuthal dependence back to twofold. This seems at odds with every other bifurcation process observed, as they all move from a lower to higher azimuthal angle dependency. This different form of bifurcation is examined in more detail in Sec. III D.

Figures 14 and 15 show the launch and return azimuthal dependencies for the seven harmonics of the GT orbit as the 

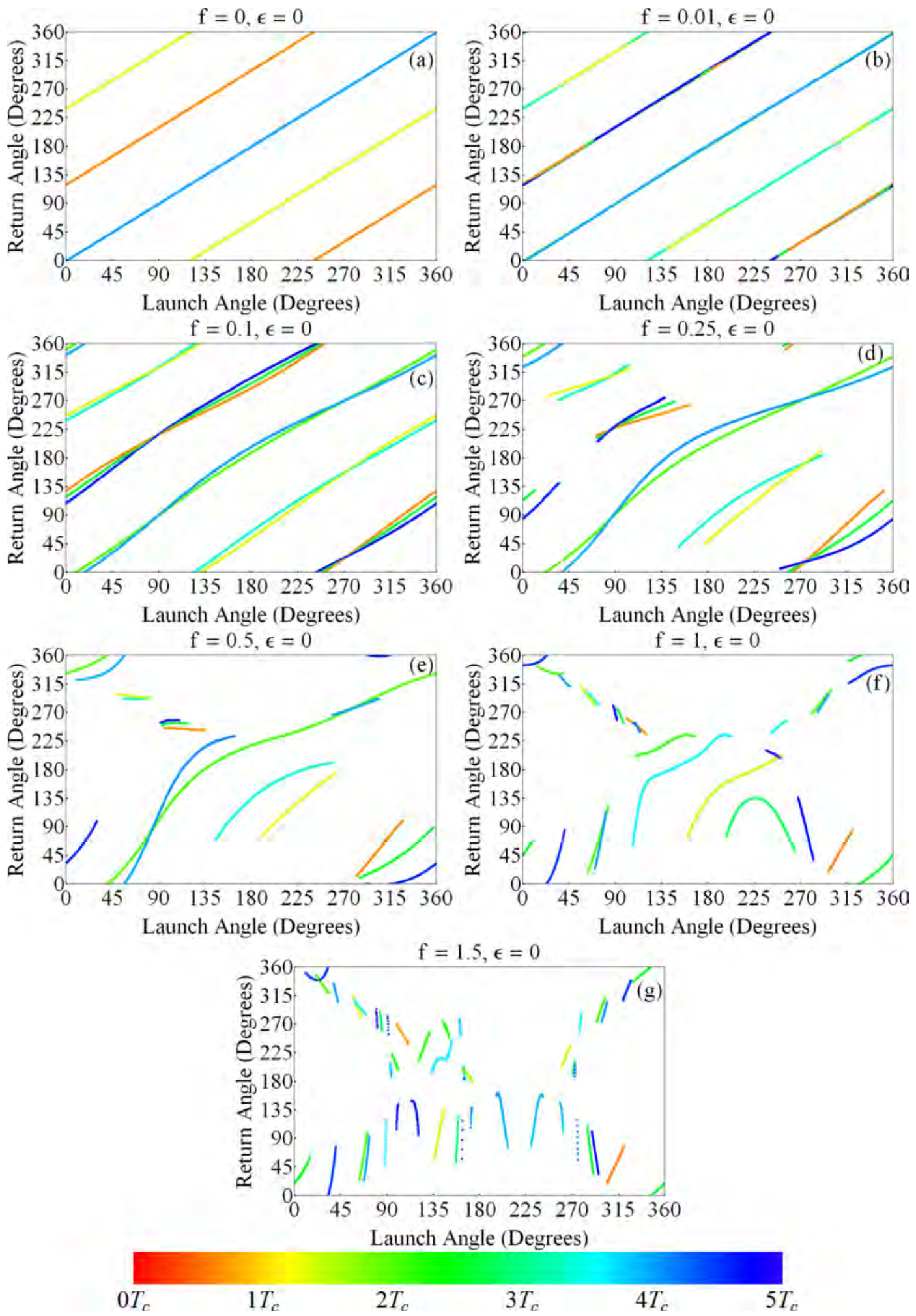

FIG. 11. Return angle as a function of launch angle for $f=0-1.5$ and $\epsilon=0$.

scaled energy is incrementally increased for a fixed scaled field of $f=0.5$. The first harmonic shows the same behavior as was observed at $f=0.25$ with the launch and return angle minima moving toward $\phi=90^{\circ}$ and $\phi=270^{\circ}$ with increasing scaled energy. The fourth harmonic once again undergoes a bifurcation moving from a twofold to fourfold dependence.
However, here the bifurcation appears at a lower scaled energy than previously seen at $f=0.25$ due to the instability the higher scaled-field induces in the azimuthal dependence. This bifurcation also evolves in the same way as can be seen when comparing figures at $f=0.25, \epsilon=4$ and $f=0.5$, $\epsilon=2$. 

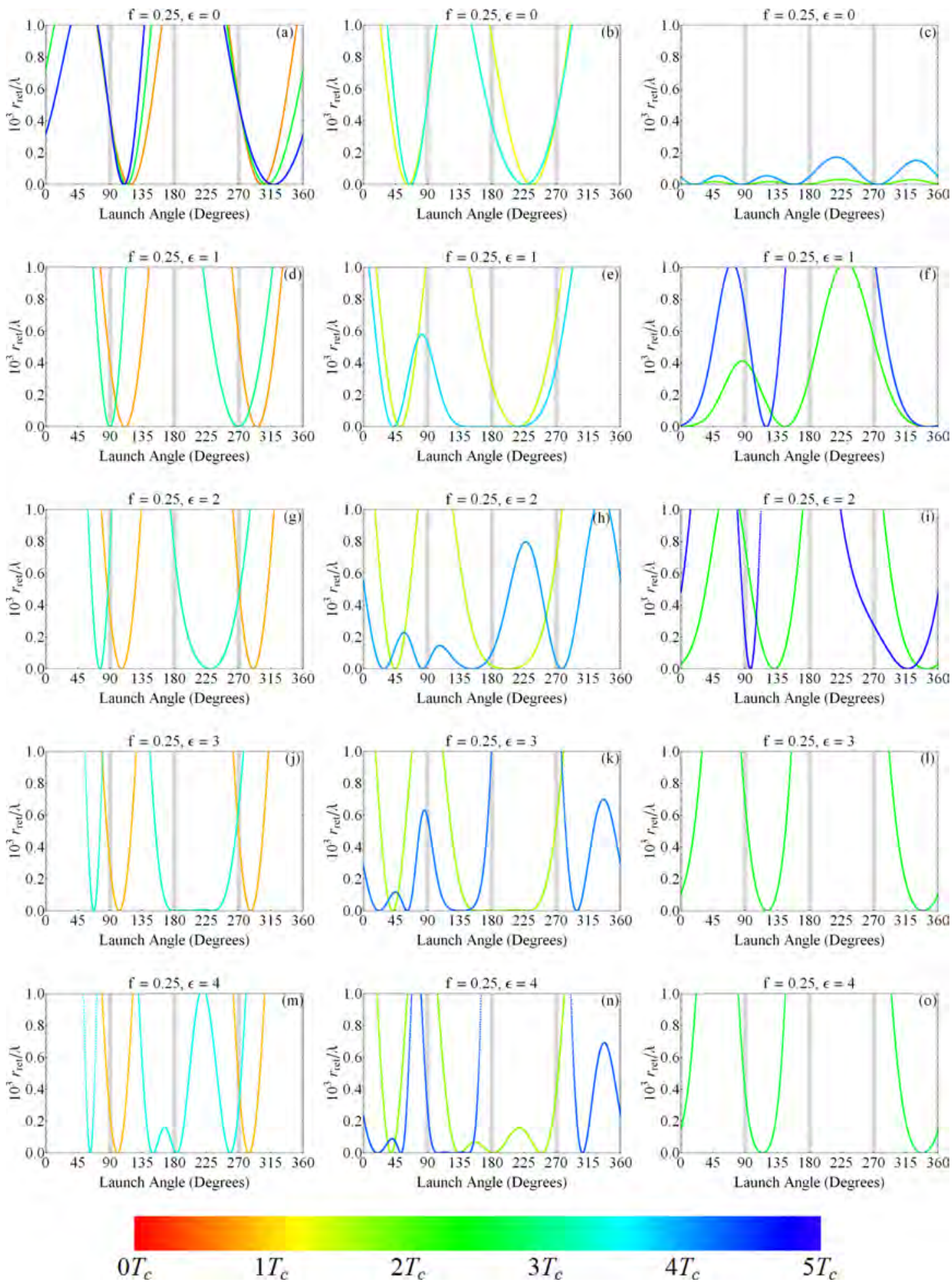

FIG. 12. Return distance $\left(r_{\text {ret }}\right)$ to the nucleus as a function of azimuthal launch angle at $f=0.25$ as the scaled energy is increased in integer increments from $\epsilon=0$ to 4 . In the figure, scaled energy increases from top to bottom. The left column shows the first, fourth, and seventh harmonics, the middle column shows the second and fifth harmonics, and the right column shows the third and sixth harmonics.

The second harmonic again moves from a twofold to fourfold dependence through a bifurcation between $\epsilon=1$ and 2. As was the case with the fourth harmonic, the second harmonic undergoes its bifurcation at a lower scaled energy with the increased scaled field. This bifurcation also evolves in the same manner at both scaled fields. The fifth harmonic starts as a twofold dependence at $\epsilon=0$ and undergoes many bifurcations and azimuthal-dependence symmetries with increasing scaled energy. The major bifurcation occurs between $\epsilon=0$ and 1 . As was the case with other harmonics discussed, this 

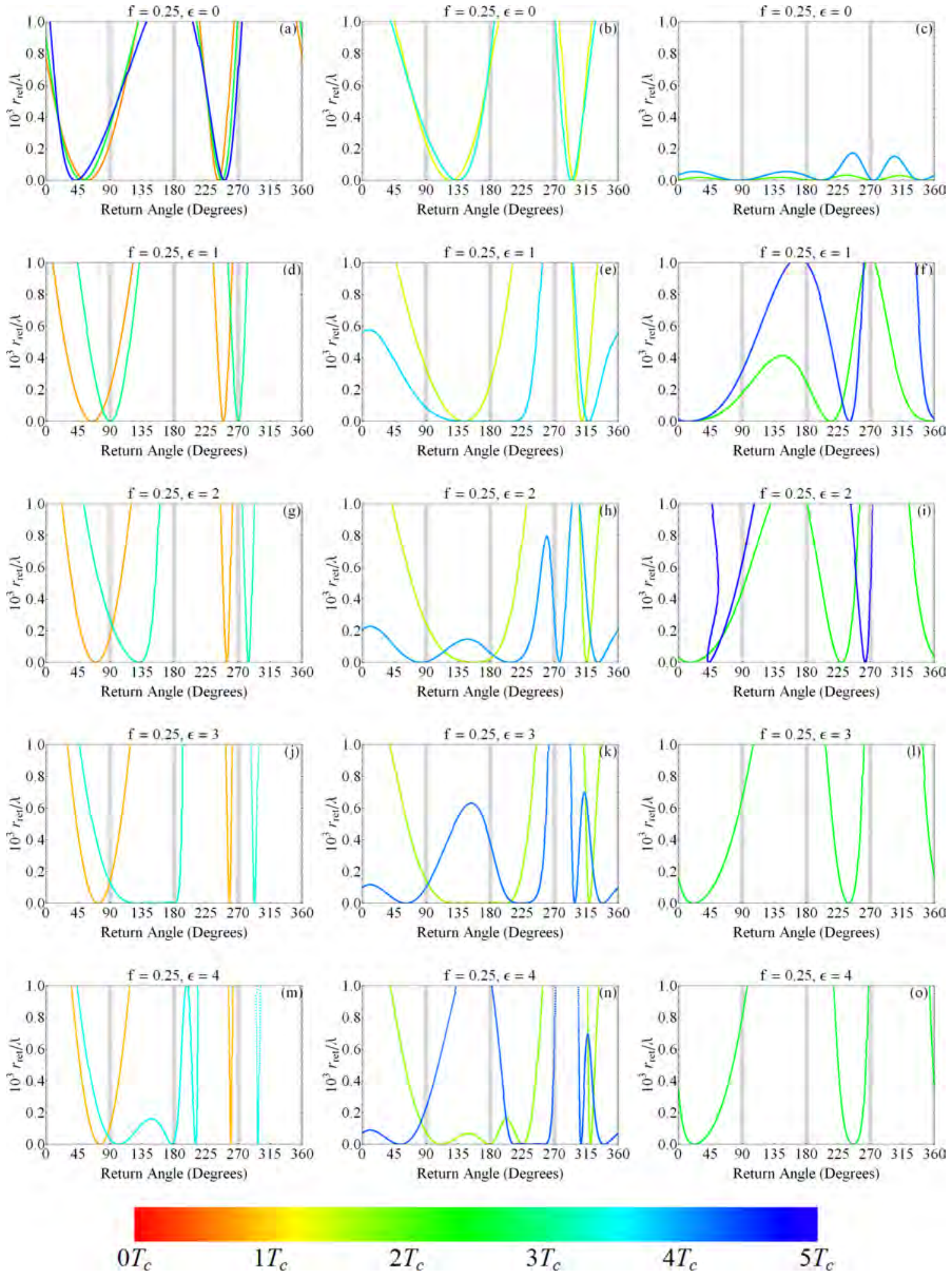

FIG. 13. Return distance $\left(r_{\text {ret }}\right)$ to the nucleus as a function of azimuthal return angle at $f=0.25$ as the scaled energy is increased in integer increments from $\epsilon=0$ to 4 . In the figure, scaled energy increases from top to bottom. The left column shows the first, fourth, and seventh harmonics, the middle column shows the second and fifth harmonics, and the right column shows the third and sixth harmonics.

bifurcation occurs at a lower scaled energy than was observed at $f=0.25$.

The third and sixth harmonics, in contrast to those discussed previously, have a much different azimuthal dependence than was observed at $f=0.25$. The shift from fourfold to twofold dependence, seen at $f=0.25$, no longer occurs. The third harmonic undergoes a new small bifurcation between $\epsilon=2$ and 3 evolving from a fourfold to sixfold azimuthal 

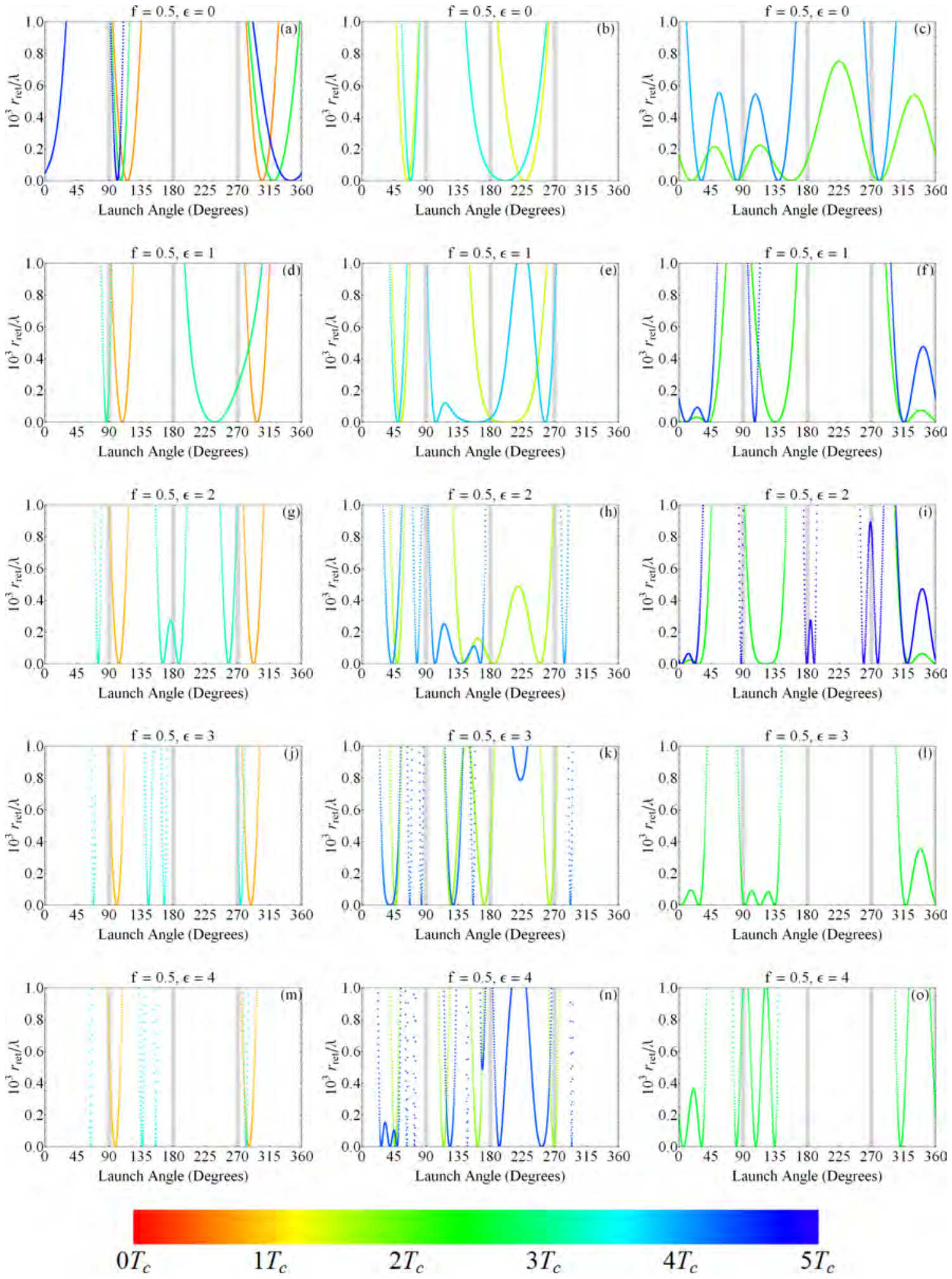

FIG. 14. Return distance $\left(r_{\text {ret }}\right)$ to the nucleus as a function of azimuthal launch angle at $f=0.5$ as the scaled energy is increased in integer increments from $\epsilon=0$ to 4 . In the figure, scaled energy increases from top to bottom. The left column shows the first, fourth, and seventh harmonics, the middle column shows the second and fifth harmonics, and the right column shows the third and sixth harmonics.

dependence. The sixth harmonic undergoes a large change between $\epsilon=1$ and 2 , with new features presenting themselves in both launch and return azimuthal angles.

\section{Bifurcation evolution with varying scaled field}

In Sec. III A, numerous bifurcations and changes in symmetry were visible in calculations when increasing the scaled field 

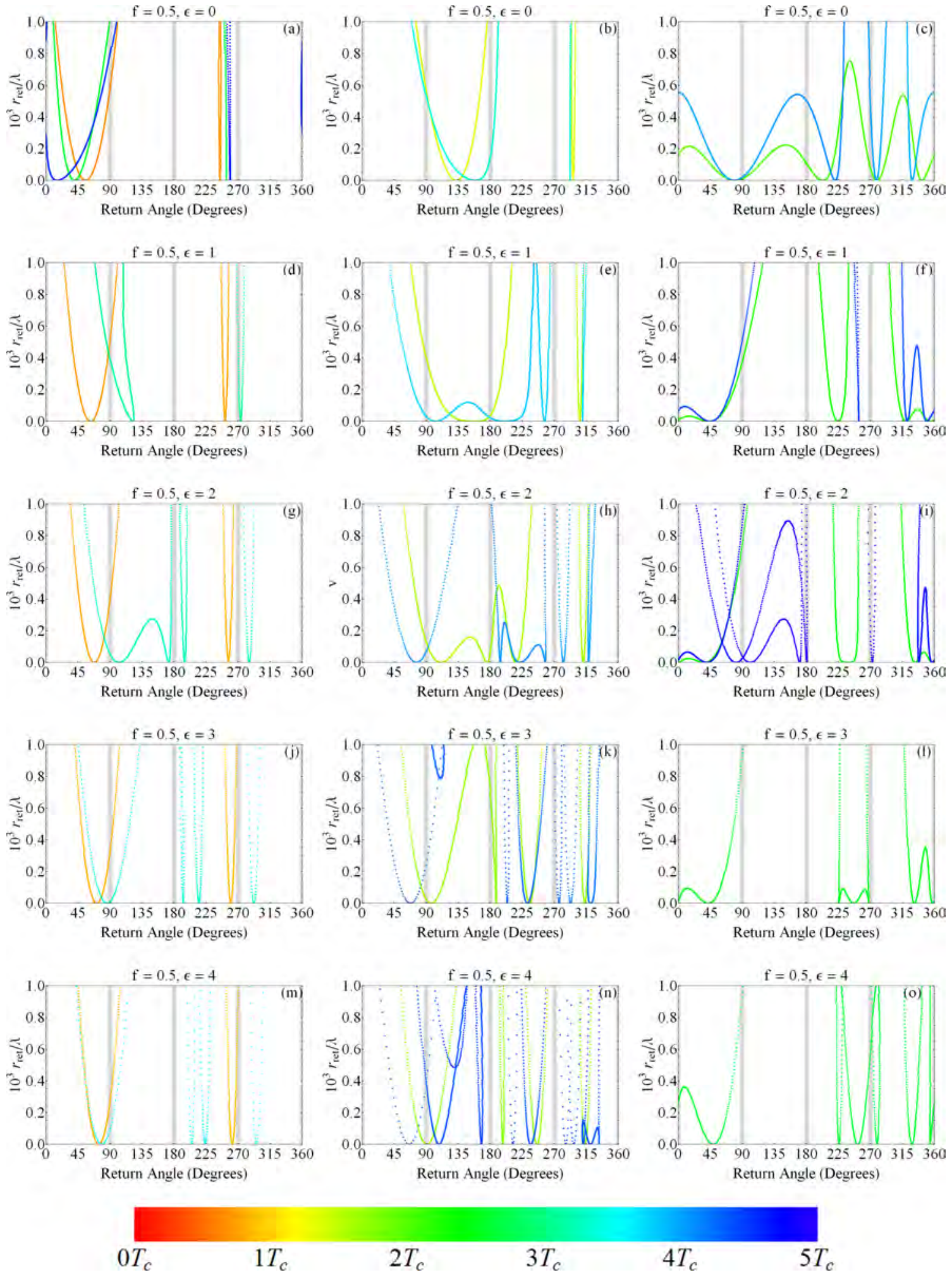

FIG. 15. Return distance $\left(r_{\text {ret }}\right)$ to the nucleus as a function of azimuthal return angle at $f=0.5$ as the scaled energy is increased in integer increments from $\epsilon=0$ to 4 . In the figure, scaled energy increases from top to bottom. The left column shows the first, fourth, and seventh harmonics, the middle column shows the second and fifth harmonics, and the right column shows the third and sixth harmonics.

from $f=0.5$ to 1 . In this section, we focus on this scaled field region in finer detail using increments of $f=0.05$ to gain a more detailed understanding of the bifurcations and azimuthal dependencies within this range.
Figures 16 and 17 show the azimuthal angle dependencies of the first, fourth, and seventh harmonics for launch and return angles respectively over this scaled-field range. The first harmonic undergoes very little change in this region. 

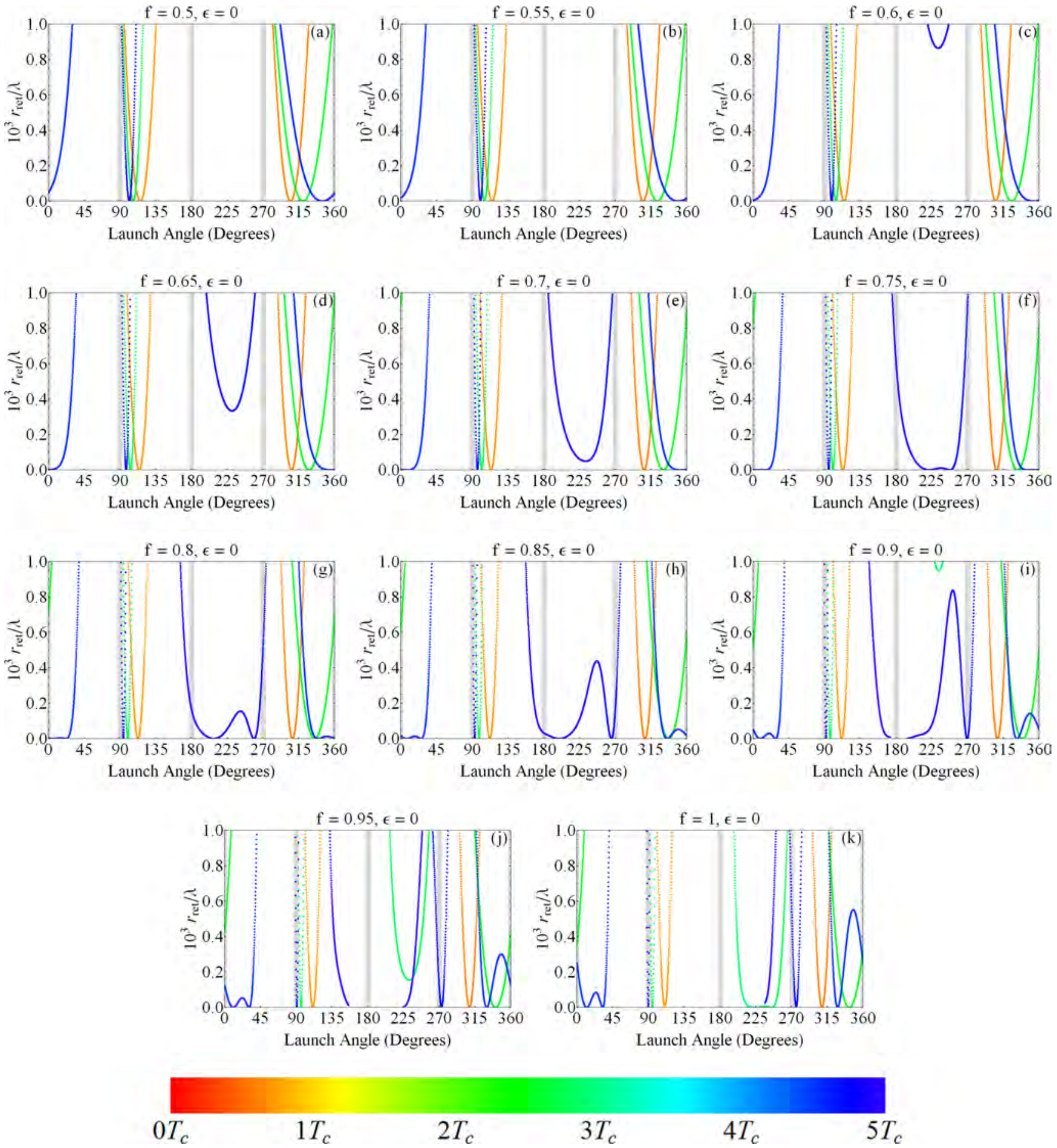

FIG. 16. Return distance $\left(r_{\text {ret }}\right)$ to the nucleus as a function of azimuthal launch angle for the first, fourth, and seventh harmonics in the scaled-field region of $f=0.5$ to 1 in steps of $f=0.05$.

The only noteworthy change coming in the orbit returning at approximately $\phi=250^{\circ}$ which increases in stability with increased scaled field over this range.

The fourth harmonic also remains relatively unchanged until $f=0.9$ where another feature begins to return close enough to the nucleus to be considered as having closed orbits. At $f=0.95$ this feature is becoming quite substantial and stable to the point that at $f=1$, it is the dominant feature in this harmonic. This feature is unusual in that the return angle azimuthal dependence is nonparabolic in nature. This was explored earlier for this particular case in Fig. 9; however, other instances do occur in the calculations for other harmonics and fields.

The seventh harmonic showed significant change in Sec. III A with another feature, a discontinuity, and the possibility of a bifurcation in the most stable of the two minima at $f=0.5$. All of these are now explained in the series of figures shown in this section. The feature comes 

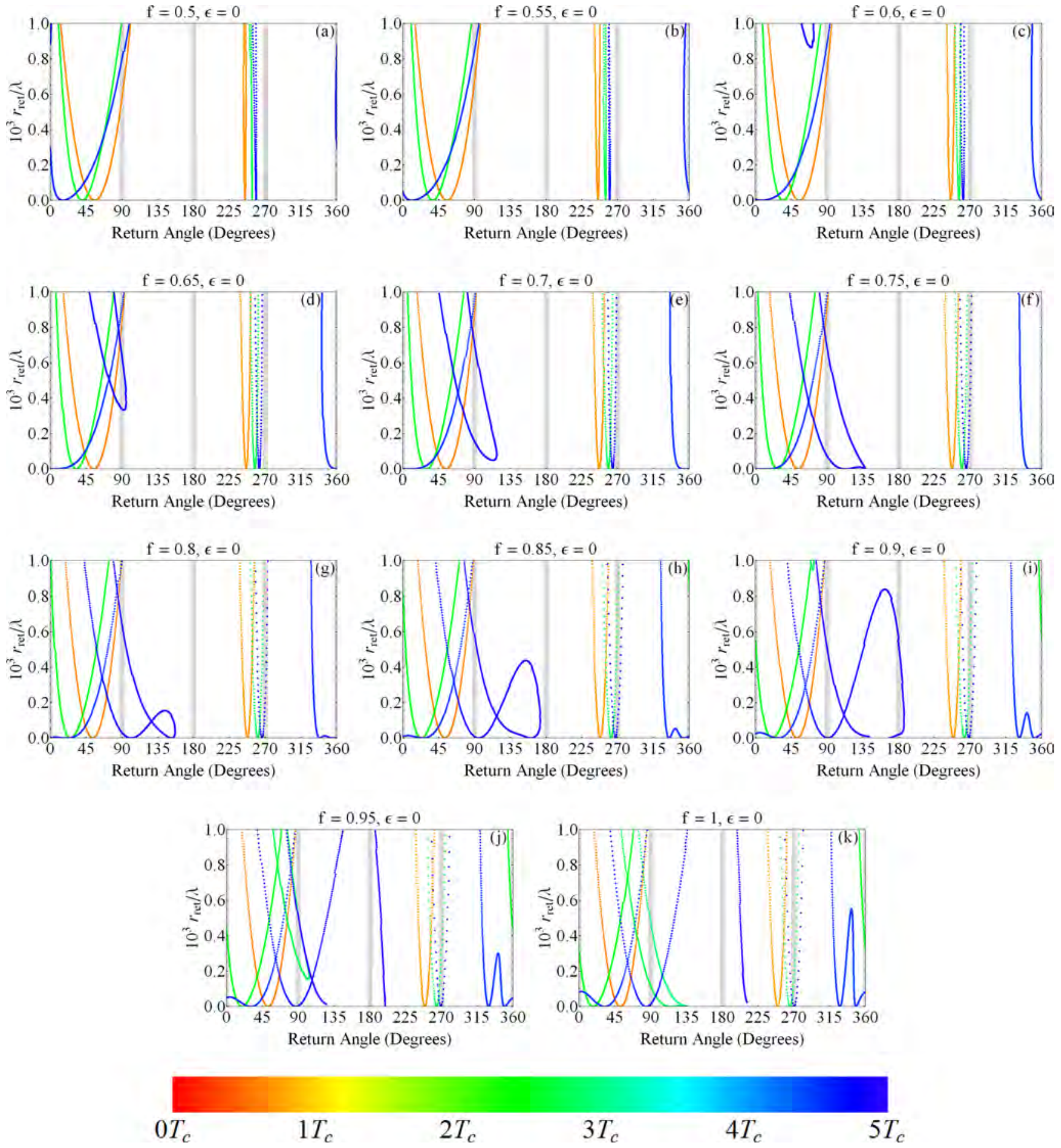

FIG. 17. Return distance $\left(r_{\mathrm{ret}}\right)$ to the nucleus as a function of azimuthal return angle for the first, fourth, and seventh harmonics in the scaled-field region of $f=0.5$ to 1 in steps of $f=0.05$.

about in much the same way as was observed in the fourth harmonic. However, here this feature begins to present itself at $f=0.6$ and is much more sensitive to variations in scaled field than the regular twofold dependence. As in the fourth harmonic, this feature presents a nonparabolic relationship in return-angle azimuthal dependence. At $f=0.9$, this feature develops a discontinuity, which is due to those missing orbits now exceeding $5 T_{c}$. These orbits would still be present in experimental data of course as this cutoff is arbitrary for numerical purposes. The most stable orbit of the two at $f=0.5$ undergoes its bifurcation from $f=0.65$ to 0.85 where a significant azimuthal angle range contains essentially closed orbits.

Figures 18 and 19 show the azimuthal angle dependencies of the second and fifth harmonics for launch and return angles, respectively, over the same scaled-field range. The 

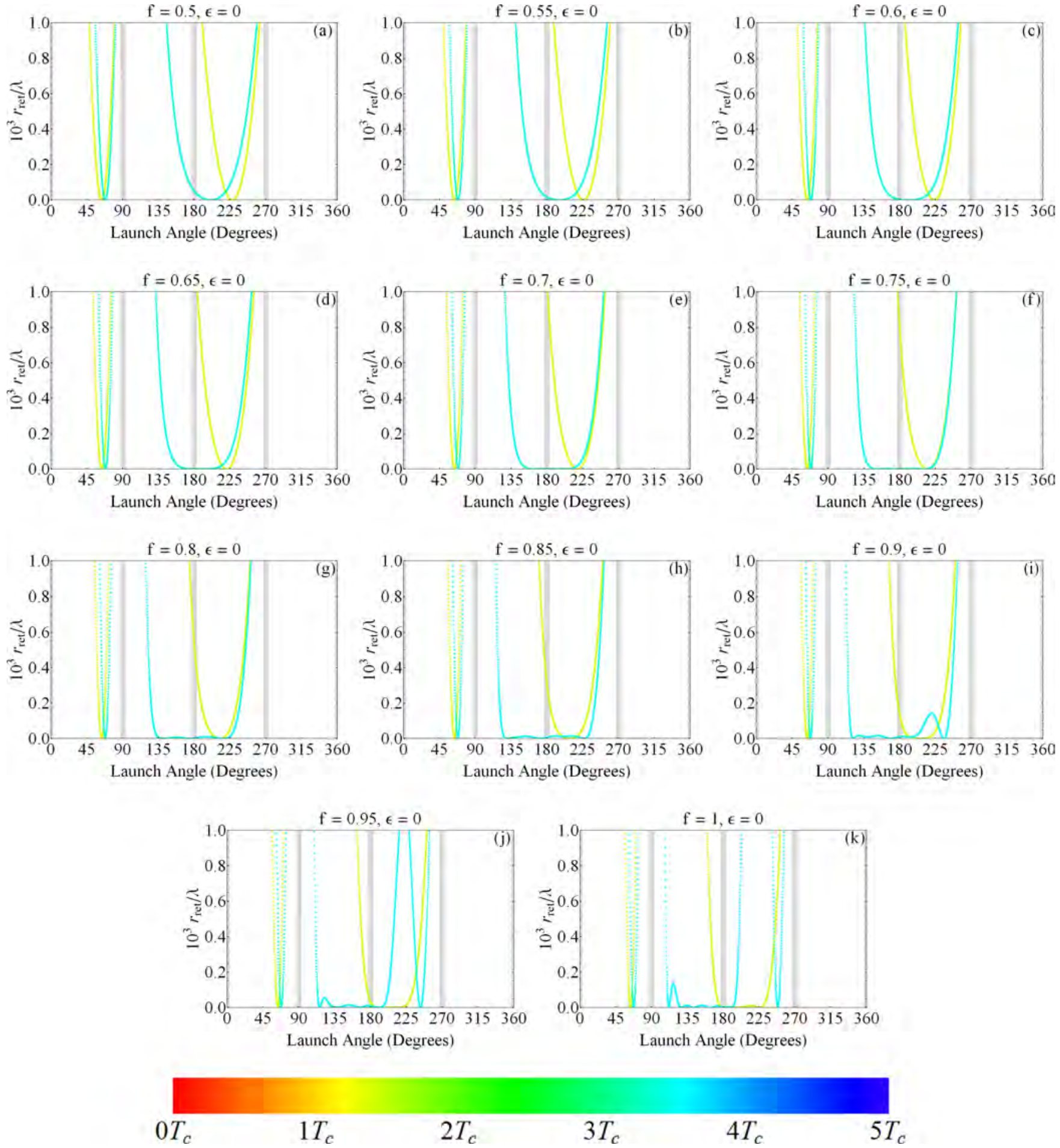

FIG. 18. Return distance $\left(r_{\text {ret }}\right)$ to the nucleus as a function of azimuthal launch angle for the second and fifth harmonics in the scaled-field region of $f=0.5$ to 1 in steps of $f=0.05$.

second harmonic, much like the first, undergoes relatively little change. However, at higher fields, the most stable of the two minima forms a wide range of closed orbits in what looks to be the beginnings of a bifurcation.

The fifth harmonic undergoes more significant change over this scaled-field range. The most stable minima in this harmonic at $f=0.5$ continues to increase in stability to the point that at $f=0.85$, almost a third of the entire azimuthal-angle space is dominated by essentially closed orbits. We should expect such a large feature to be apparent in experimental data. At higher scaled fields this large area undergoes multiple bifurcations; however, the area around $\phi=180^{\circ}$ remains very stable in both launch and return angles.

Figures 20 and 21 show the azimuthal angle dependencies of the third and sixth harmonics for launch and return angles respectively over the same scaled-field range. Both 

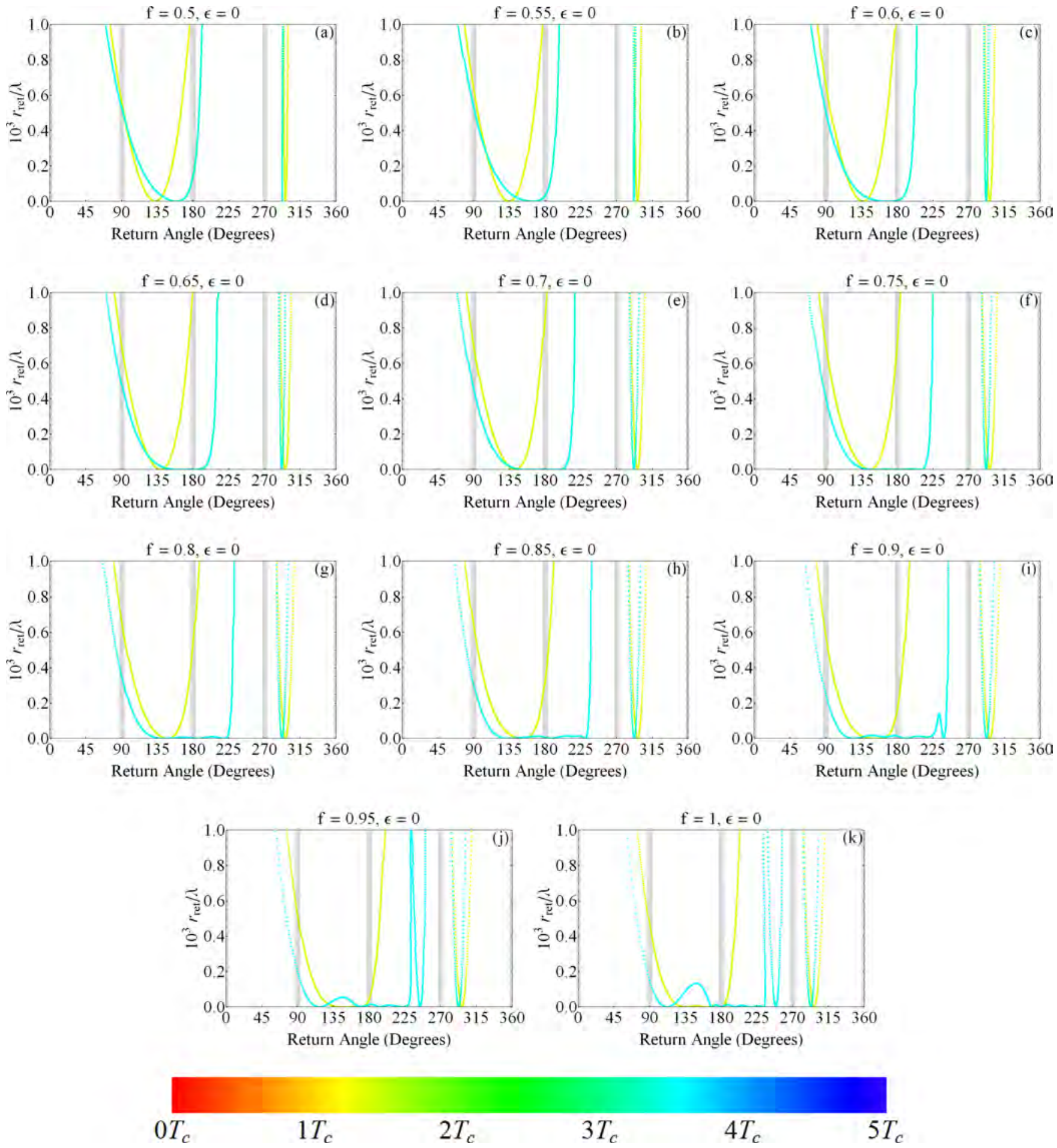

FIG. 19. Return distance $\left(r_{\text {ret }}\right)$ to the nucleus as a function of azimuthal return angle for the second and fifth harmonics in the scaled-field region of $f=0.5$ to 1 in steps of $f=0.05$.

harmonics retain their fourfold azimuthal dependence over this scaled-field range. As the scaled field is increased, the orbits in both harmonics become less stable as seen by the increasing curvature of the parabolas in the calculations. The third harmonic begins a small bifurcation process at the higher end of this scaled-field region; however, it is unlikely this would be significant in any experimental results.

\section{Reduction of azimuthal dependence in third and sixth harmonics}

In Sec. III B, we observed that in Figs. 12 and 13, the third and sixth harmonics moved from a fourfold to twofold azimuthal dependence through a bifurcation process when the energy was increased from $\epsilon=0$ to 1 . This was unexpected as every other form of azimuthal symmetry change we have 

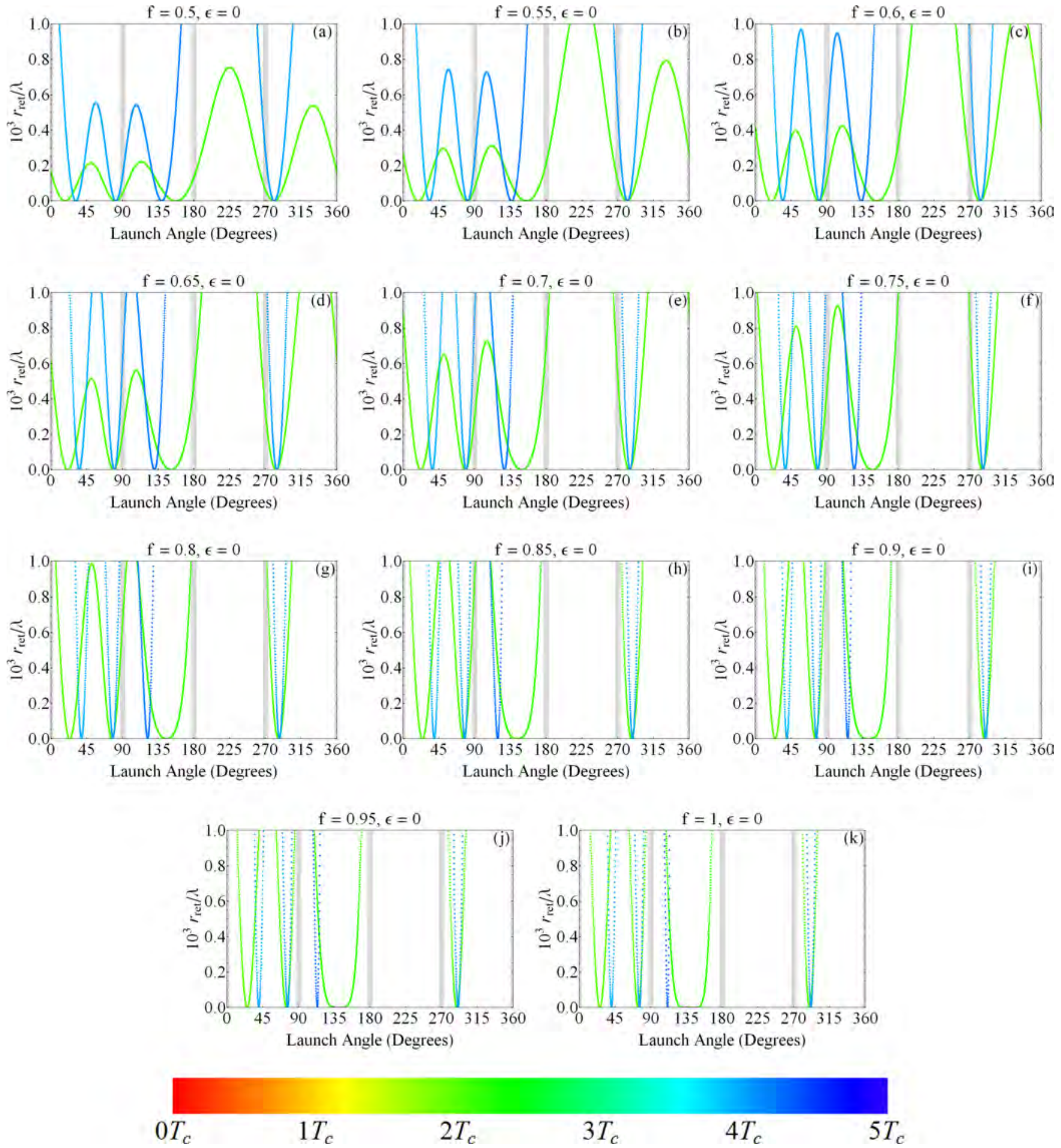

FIG. 20. Return distance $\left(r_{\text {ret }}\right)$ to the nucleus as a function of azimuthal launch angle for the third and sixth harmonics in the scaled-field region of $f=0.5$ to 1 in steps of $f=0.05$.

observed involved moving from a lower to higher azimuthal angle dependency. In this section, we revisit this feature using a finer scaled-energy mesh of $\Delta \epsilon=0.1$ to gain a better insight into this different form of bifurcation.

Figures 22 and 23 show the launch and return azimuthal angle dependencies, respectively, for the third and sixth harmonics at $f=0.25$ and scaled energies ranging from $\epsilon=0$ to 1 in steps of 0.1 . We observe that as the scaled energy is increased from $\epsilon=0$ to 0.1 , the relative height of the peaks in the azimuthal dependence changes substantially. These harmonics appear to be much more sensitive to variations in scaled energy than scaled field. By increasing the scaled energy to $\epsilon=0.3$, the two peaks either side of the external electric-field orientation at $\phi=0^{\circ}$ have nearly disappeared 

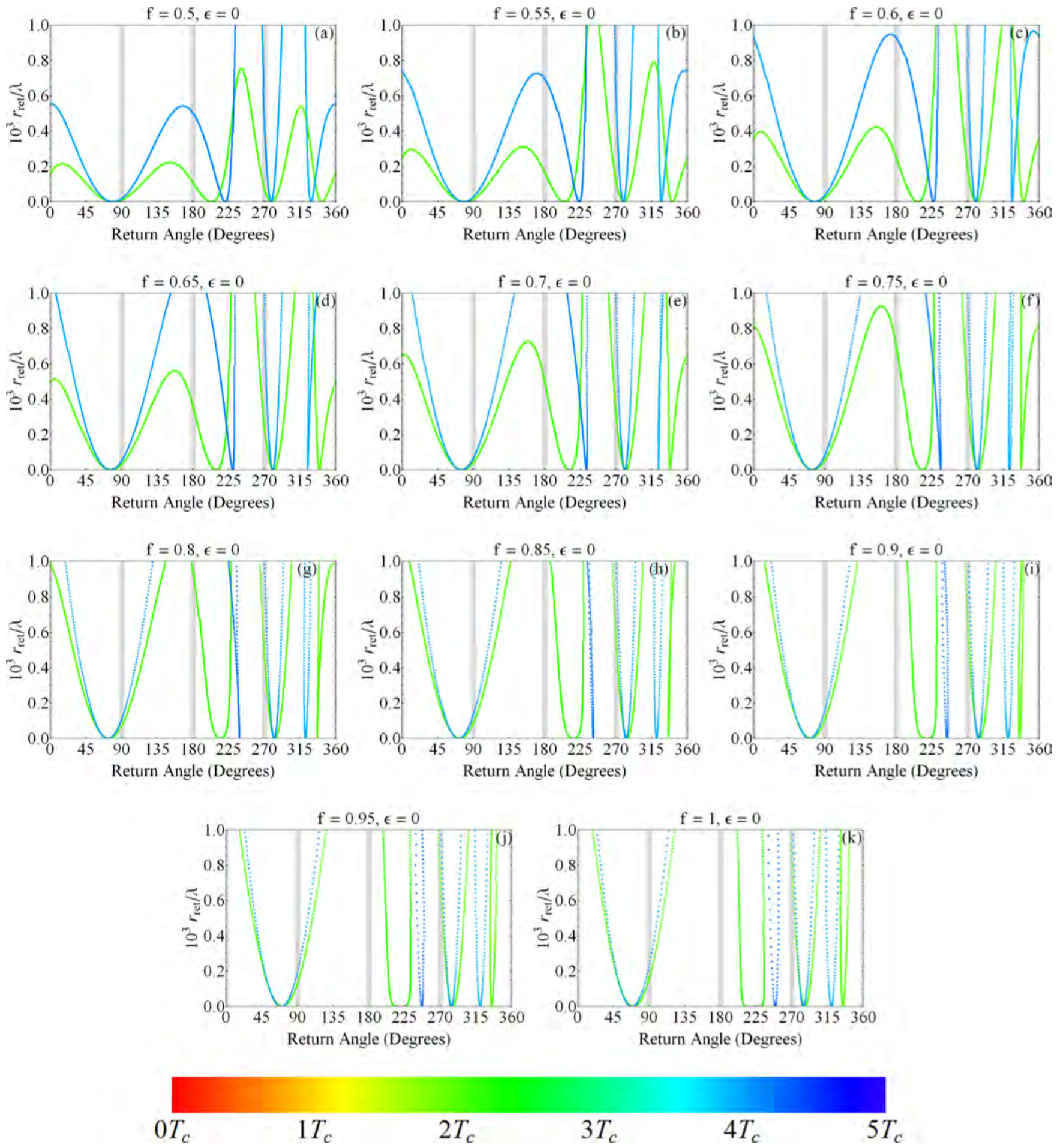

FIG. 21. Return distance $\left(r_{\text {ret }}\right)$ to the nucleus as a function of azimuthal return angle for the third and sixth harmonics in the scaled-field region of $f=0.5$ to 1 in steps of $f=0.05$.

completely. This creates a large azimuthal area of closed orbits as the scaled energy is incrementally increased, which encompasses the external electric field orientation at $\phi=0^{\circ}$ in both launch and return angles.

\section{CONCLUSION}

The azimuthal dependence of the first seven GartonTomkins orbit harmonics have been investigated thoroughly in crossed magnetic and electric fields. In cylindrically symmetric external-field geometries, this azimuthal dependence is absent. However, in the crossed-fields geometry, due to the breaking of the rotational symmetry around the magnetic-field axis, this is no longer the case and the azimuthal angle dependence must be considered. In this work, we focused on the isotropic case which is relevant to both atomic systems and isotropic semiconductors. However, we believe these results to 

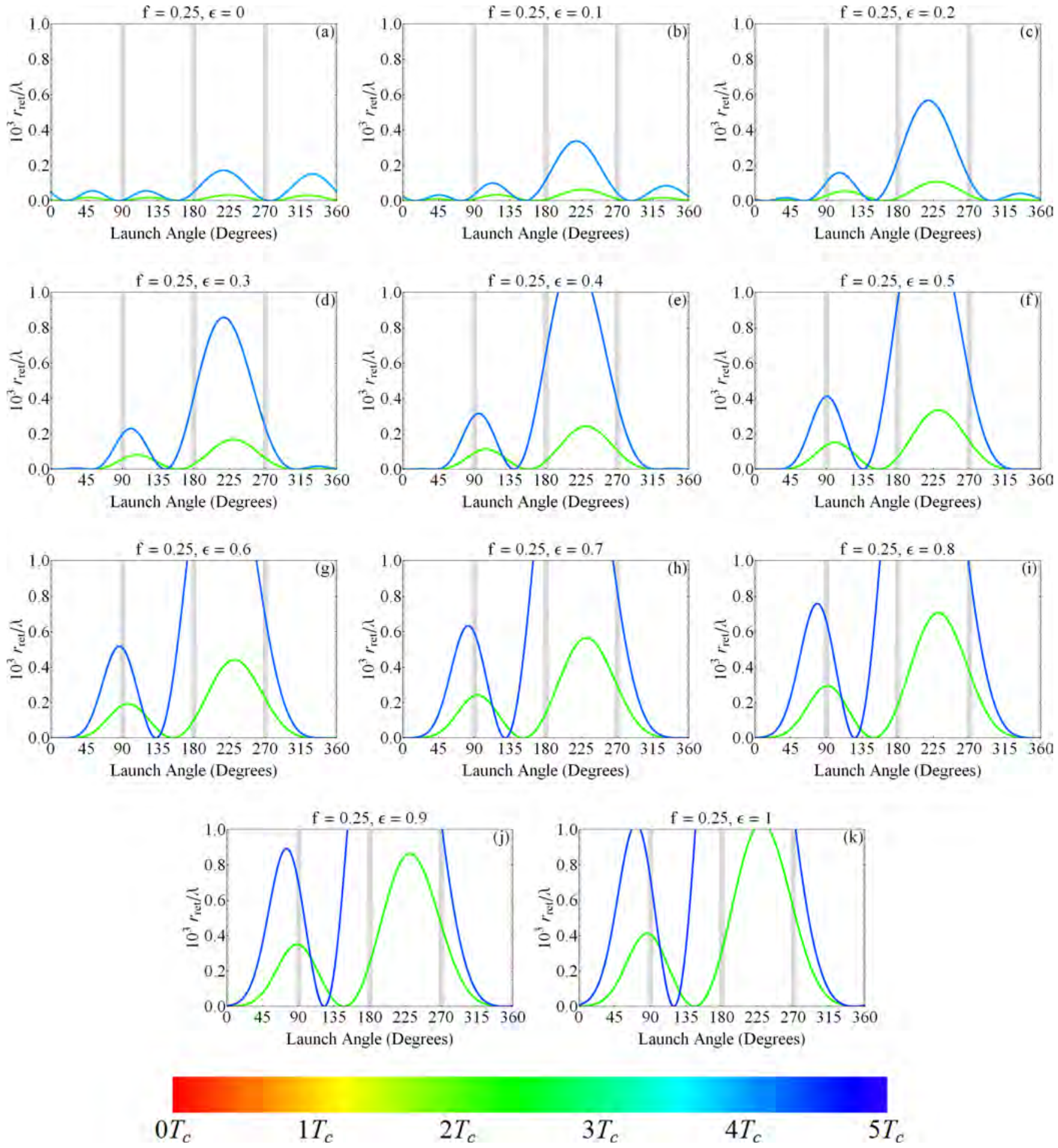

FIG. 22. Return distance $\left(r_{\text {ret }}\right)$ to the nucleus as a function of azimuthal launch angle for the third and sixth harmonics at $f=0.25$ for varying scaled energy between $\epsilon=0$ and 1 in 0.1 steps.

also be applicable to a specific case in the sixfold conduction band semiconductor environment of silicon, where the magnetic field is aligned along the (001) crystallographic axis and the electric field is aligned along the (100) crystallographic axis in the perpendicular plane. We found that by introducing a weak electric field perpendicular to the applied magnetic field, the Garton-Tomkins orbit and its harmonics which lack rotational symmetry about the magnetic-field axis show twofold azimuthal dependence. However, for harmonics which are essentially periodic, namely the third and sixth harmonics, a fourfold azimuthal dependence was observed. These rotationally symmetric harmonics were also more stable against increases in scaled field. We also found that the symmetries of the Garton-Tomkins orbit, and its harmonics, were more sensitive to variations in scaled energy than scaled field. 

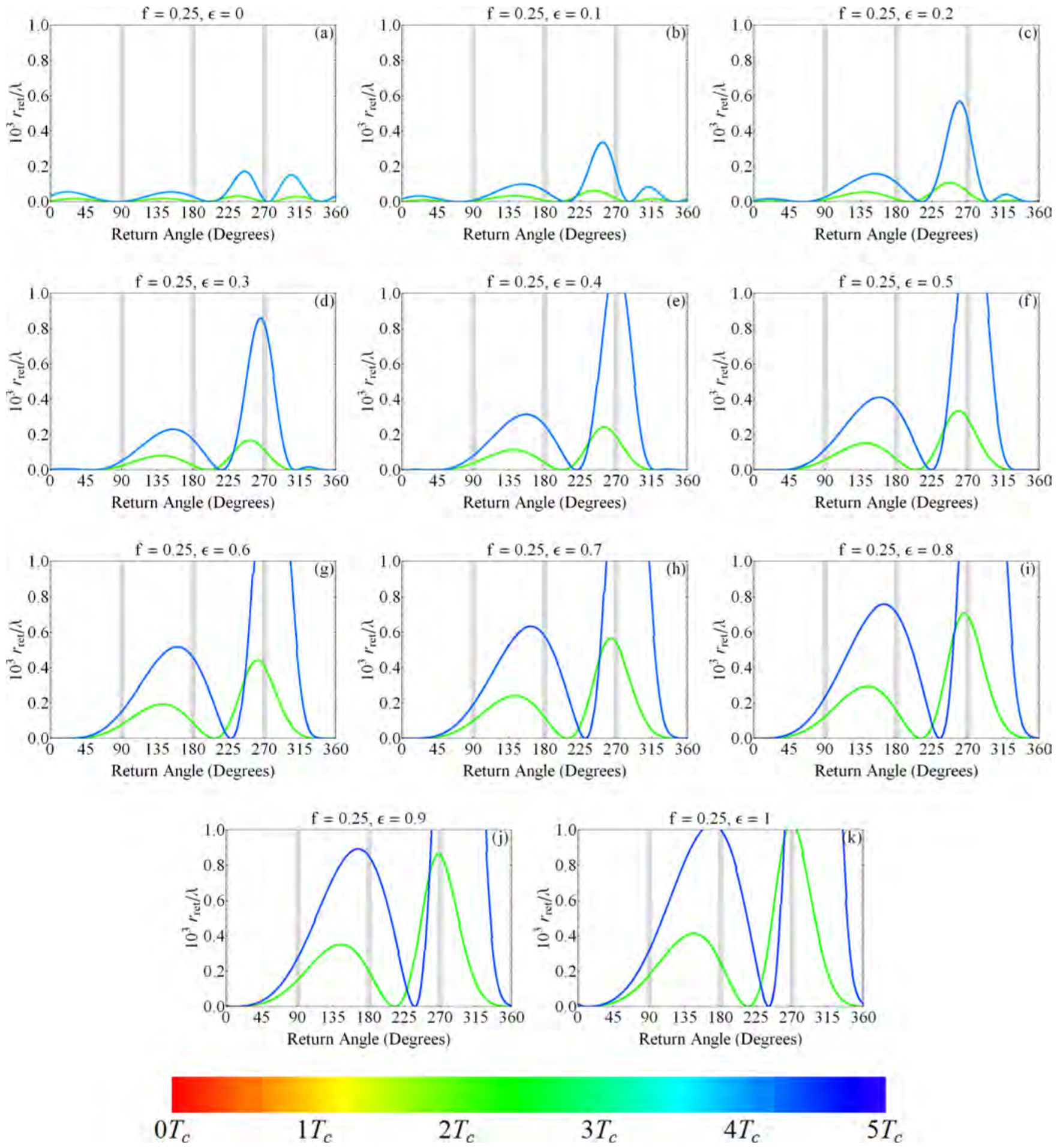

FIG. 23. Return distance $\left(r_{\text {ret }}\right)$ to the nucleus as a function of azimuthal return angle for the third and sixth harmonics at $f=0.25$ for varying scaled energy between $\epsilon=0$ and 1 in 0.1 steps.

In the course of our calculations, many bifurcation processes were observed where a continuum of essentially closed orbits were present over up to a third of the entire azimuthal angle range. As is the case with bifurcations in other field configurations, we expect these regions to present large resonances in experimental data. We also found here the possibility of a bifurcation reducing the azimuthal angle dependence of the third and sixth harmonics at $f=0.25$ and small positive scaled energies. This is counter to all other bifurcation processes which were observed in our investigation.

\section{ACKNOWLEDGMENT}

The work was supported by the Australian Research Council and the University of Wollongong (under the UIC International Links Scheme). 
[1] W. R. S. Garton and F. S. Tomkins, Astrophys. J. 158, 839 (1969).

[2] J. A. C. Gallas and R. F. O'Connell, J. Phys. B 15, L75 (1982).

[3] A. Holle, G. Wiebusch, J. Main, B. Hager, H. Rottke, and K. H. Welge, Phys. Rev. Lett. 56, 2594 (1986).

[4] J. Main, G. Wiebusch, A. Holle, and K. H. Welge, Phys. Rev. Lett. 57, 2789 (1986).

[5] D. Wintgen, J. Phys. B 20, L511 (1987).

[6] D. Wintgen and H. Friedrich, Phys. Rev. A 36, 131 (1987).

[7] M. L. Du and J. B. Delos, Phys. Rev. A 38, 1896 (1988).

[8] A. Holle, J. Main, G. Wiebusch, H. Rottke, and K. H. Welge, Phys. Rev. Lett. 61, 161 (1988).

[9] H. Friedrich and H. Wintgen, Phys. Rep. 183, 37 (1989).

[10] D. Delande, Phys. Scr. T34, 52 (1991).

[11] A. König, J. Neukammer, K. Vietzke, M. Kohl, H. J. Grabka, H. Hieronymus, and H. Rinneberg, Phys. Rev. A 38, 547 (1988).

[12] M. A. Iken, F. Borondo, R. M. Benito, and T. Uzer, Phys. Rev. A 49, 2734 (1994).

[13] H. H. Fielding, J. Wals, W. J. van der Zande, and H. B. van Linden van den Heuvell, Phys. Rev. A 51, 611 (1995).

[14] M. W. Beims and J. A. C. Gallas, Phys. Rev. A 62, 043410 (2000).

[15] T. Topçu and F. Robicheaux, J. Phys. B 40, 1925 (2007).

[16] G. Wiebusch, J. Main, K. Krüger, H. Rottke, A. Holle, and K. H. Welge, Phys. Rev. Lett. 62, 2821 (1989).

[17] J. von Milczewski and T. Uzer, Phys. Rev. E 55, 6540 (1997).
[18] C. Neumann, R. Ubert, S. Freund, E. Flöthmann, B. Sheehy, K. H. Welge, M. R. Haggerty, and J. B. Delos, Phys. Rev. Lett. 78, 4705 (1997).

[19] J. Rao, D. Delande, and K. T. Taylor, J. Phys. B 34, L391 (2001).

[20] J. Rao and K. T. Taylor, J. Phys. B 35, 2627 (2002).

[21] S. Freund, R. Ubert, E. Flöthmann, K. Welge, D. M. Wang, and J. B. Delos, Phys. Rev. A 65, 053408 (2002).

[22] T. Bartsch, J. Main, and G. Wunner, Phys. Rev. A 67, 063410 (2003).

[23] T. Bartsch, J. Main, and G. Wunner, Phys. Rev. A 67, 063411 (2003).

[24] F. Schweiner, J. Main, H. Cartarius, and G. Wunner, Phys. Rev. E 91, 012915 (2015).

[25] R. R. Freeman, N. P. Economou, G. C. Bjorklund, and K. T. Lu, Phys. Rev. Lett. 41, 1463 (1978).

[26] M. Courtney, N. Spellmeyer, H. Jiao, and D. Kleppner, Phys. Rev. A 51, 3604 (1995).

[27] M. Courtney, H. Jiao, N. Spellmeyer, D. Kleppner, J. Gao, and J. B. Delos, Phys. Rev. Lett. 74, 1538 (1995).

[28] C. Bleasdale, A. Bruno-Alfonso, and R. A. Lewis, Phys. Rev. A 93, 023405 (2016).

[29] Z. Chen, W. Zhou, B. Zhang, C. H. Yu, J. Zhu, W. Lu, and S. C. Shen, Phys. Rev. Lett. 102, 244103 (2009).

[30] A. Bruno-Alfonso, C. Bleasdale, G. V. B. de Souza, and R. A. Lewis, Phys. Rev. A 89, 043425 (2014).

[31] C. Bleasdale and R. A. Lewis, Phys. Status Solidi B 252, 2657 (2015). 